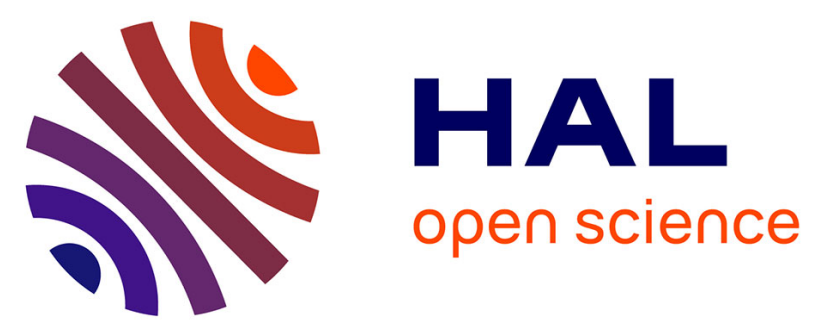

\title{
An algebraic continuous time parameter estimation for a sum of sinusoidal waveform signals
}

\author{
Rosane Ushirobira, Wilfrid Perruquetti, Mamadou Mboup
}

\section{To cite this version:}

Rosane Ushirobira, Wilfrid Perruquetti, Mamadou Mboup. An algebraic continuous time parameter estimation for a sum of sinusoidal waveform signals. International Journal of Adaptive Control and Signal Processing, 2016, 30 (12), pp.1689-1713. 10.1002/acs.2688 . hal-01342210

\section{HAL Id: hal-01342210 \\ https://inria.hal.science/hal-01342210}

Submitted on 25 Aug 2016

HAL is a multi-disciplinary open access archive for the deposit and dissemination of scientific research documents, whether they are published or not. The documents may come from teaching and research institutions in France or abroad, or from public or private research centers.
L'archive ouverte pluridisciplinaire HAL, est destinée au dépôt et à la diffusion de documents scientifiques de niveau recherche, publiés ou non, émanant des établissements d'enseignement et de recherche français ou étrangers, des laboratoires publics ou privés. 


\title{
An algebraic continuous time parameter estimation for a sum of sinusoidal waveform signals
}

\author{
Rosane Ushirobira, Wilfrid Perruquetti and Mamadou Mboup
}

\begin{abstract}
In this paper, a novel algebraic method is proposed to estimate amplitudes, frequencies and phases of a biased and noisy sum of complex exponential sinusoidal signals. The resulting parameter estimates are given by original closed formulas, constructed as integrals acting as time-varying filters of the noisy measured signal. The proposed algebraic method provides faster and more robust results, compared to usual procedures. Some computer simulations illustrate the efficiency of our method.
\end{abstract}

\section{Index Terms}

Parameter identification; Differential Algebra; Sinusoidal wave; Noise

\section{CONTENTS}

I Introduction

II Notations 23

III Problem formulation $\quad 3$

IV Annihilators 5

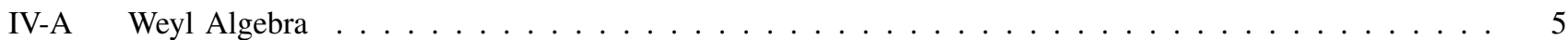

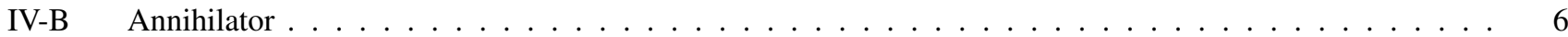

V Parameter estimation $\quad \ldots$

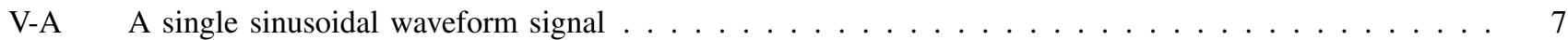

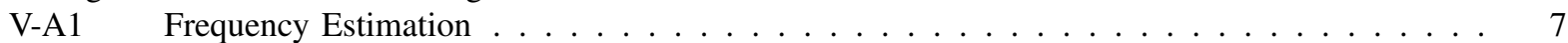

V-A2 Amplitude and phase estimation $\ldots \ldots \ldots \ldots \ldots \ldots$

V-B A sum of two sinusoidal waveform signals $\ldots \ldots \ldots \ldots \ldots \ldots \ldots$

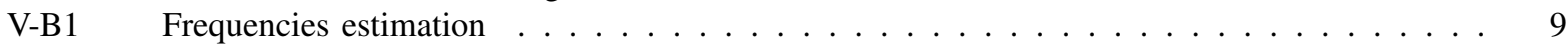

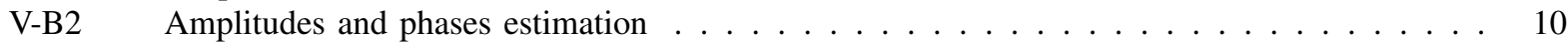

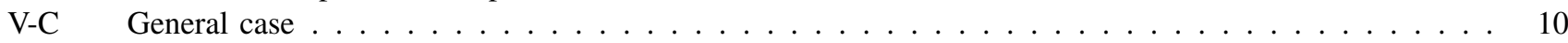

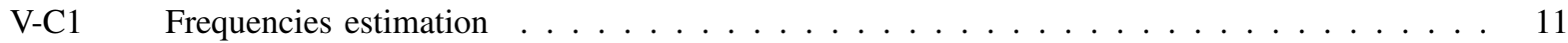

$\mathrm{V}-\mathrm{C} 2 \quad$ Amplitudes and phases estimation $\ldots \ldots \ldots \ldots \ldots \ldots \ldots$

$\begin{array}{llr}\text { VI Simulations } & 15\end{array}$

$\begin{array}{ll}\text { References } & 15\end{array}$

Appendix

A Proof of Lemma $2 \ldots \ldots \ldots$

B Proof of theorem $1 \ldots \ldots \ldots$

Rosane Ushirobira is with Inria, Non-A team, Villeneuve d'Ascq, France \& Institut de Mathématiques de Bourgogne (CNRS), Université de Bourgogne (e-mail: Rosane.Ushirobira@inria.fr).

Wilfrid Perruquetti is with École Centrale de Lille \& CRIStAL (CNRS), France \& Inria, Non-A team, Villeneuve d'Ascq, France (e-mail: wilfrid.perruquettieinria.fr).

Mamadou Mboup is with CReSTIC, Université de Reims Champagne Ardenne, France \& Inria, Non-A team, Villeneuve d'Ascq, France (e-mail: Mamadou.Mboup@univ-reims.fr). 


\section{INTRODUCTION}

Parameter estimation of a biased sum of sinusoidal waveform signals in a noisy environment is an important issue that occurs in many practical engineering problems such as:

- communications: e.g. signal demodulation [1], [2]; pitch perception in sounds [3].

- power system, e.g. the fundamental frequency reflecting the dynamic energy balance between load and generating power, must be obtained in a fraction of the period in the presence of harmonics and noise (see [4], [5], [6]); regulation of electronic power converters [7].

- bio-medics, e.g. electromyography (EMG) [8]; circadian rhythm of biological cells [9].

- mechanics, e.g. modal identification for flexible structures [10]; closed-loop identification method combined with an outputfeedback controller of an uncertain flexible robotic arm [11]; vibration reduction in helicopter [12]; in disk drive [13]; in magnetic bearings [14].

The above list is far from being exhaustive. An additional motivating and rather unusual example is given by the posture estimation of a human body in the sagittal plane using only accelerometer measurements. It may seem odd to try to recover the position using accelerometer measurements, however thanks to the quasi-periodicity of the movement, this study can be reduced to the parameter estimation of a sum of three sinusoidal waveform signals, see [15] for details.

To formalize our parameter estimation problem, let us consider a finite sum of complex exponential functions:

$$
x(t)=\sum_{k=1}^{n} \alpha_{k} \mathrm{e}^{i\left(\omega_{k} t+\phi_{k}\right)},
$$

where $\alpha_{k}$ denotes the amplitude, $\omega_{k}$ the frequency and $\phi_{k}$ the phase, for each $1 \leq k \leq n$. The signal $x(t)$ has to be recovered or estimated from the biased and noisy output measure

$$
y(t)=x(t)+\beta+\varpi(t),
$$

where $\beta$ is an unknown constant bias and $\varpi(t)$ is a noise, also complex valued. More precisely, the parameter estimation problem for $x(t)$ consists in estimating the triplets (amplitude, frequency, phase), that is, $\left(\alpha_{k}, \omega_{k}, \phi_{k}\right)$ for all $k$ and for a sum of an unknown number of complex sinusoidal functions ( $n$ is not a priori known). This problem was notably examined by G. Riche de Prony in his 1795 seminal paper [16] (see also [17], [18] for more modern approaches).

To solve this parameter estimation problem, many different methods have been developed (see [19], [20] for surveys), such as linear regression [21], [18], the adaptive least square method [22], subspace methods (high resolution) [23], [24], [17], the extended Kalman filter introduced in [25], [26] and refined in [27] where a simple tuning rule is given, the notches filter introduced simultaneously in [28] and [29] giving biased estimates of the frequency for standard notch (see [30]) with a first improvement obtained in [31] and an adaptive version in [32] (see also [33]), adaptive sogi-filters [34], techniques borrowed from adaptive nonlinear control [35], [36] or alternatively [37], [38] and more recently [39], [40], [41], [42]. The relation between elementary symmetric functions on frequencies of multi-sine wave signals and its multiple integrals has been also investigated on [43] allowing interesting estimation approaches of the frequencies.

In this work, a novel algebraic method is developed to provide estimates for all amplitudes, phases and frequencies of the noisy biased signal $y(t)$. One of the main advantages of considering this parameter estimation problem within our algebraic framework is to provide closed formulas for all the estimates. This has not been yet proposed in the existing literature and it consists in a real benefit of our estimation method. An important issue in this algebraic approach is that it relies heavily on differential elimination. So, for a given estimation problem, many different estimators can be devised, depending on which annihilators are used to eliminate the undesired terms in the algebraic operational expressions. We refer to [44] where this is well illustrated through a change-point detection problem. Now, it appears that the quality of an estimator varies markedly with the order of the selected annihilators. The Weyl Algebra point of view that we introduce here within the algebraic approach allows one to characterize and select the minimal order annihilators associated to any given estimation problem. This is a second main advantage of the present paper. Moreover, all above mentioned results (with exception of [19], [21], [34] that need half of the period to recover the parameters, [10] that uses also algebraic techniques for a single sinusoidal and [45] by classical methods) deal only with the frequencies estimation problem, while our method allows the estimation of all parameters, including amplitudes and phases. Furthermore, let us stress that only frequencies are estimated in [42]. M. Hou estimates phases, frequencies and amplitudes using adaptive identifiers in [45] and the simulated examples within do provide fast estimates, however in more than a fraction of the period. Our simulation show that estimates can be obtained faster than this last approach. Nevertheless, the estimation of these parameters in a fraction of the time signal, in a robust manner, in the presence of noise and an unknown constant bias, is not yet fully resolved.

This paper draws its inspiration from the algebraic analysis of [46] (that provides an algebraic framework for parameter identification in linear systems), [47] (where some signal processing paradigms are investigated), [48] (that provides compression techniques within this algebraic support), [49], [50], [51], [52], [53]. In addition to numerical simulations found in these papers, we refer to [54], [55], [56], [57], [58], [59] for application to numerical differentiation in noisy environment using this algebraic setting and to [2], [60], [10], [61], [62], [63], [11], [64], [65] for some more concrete and encouraging applications. Concerning 
our parameter estimation problem (Prony's problem), earlier works use algebraic approaches. For instance, in [60] a particular algebraic solution was obtained for a single sinusoidal signal and compared with other techniques, carrying out an analysis of robustness as well. At the same time, the proposed result was extended to the case of damping sinusoidal signals in [10] and [61]: those results were combined with a controller and experimentally tested on an uncertain flexible robotic arm (see [66], [11]). This technique was extended to the sum of two sinusoidal signals [62] and the obtained results were based on somewhat ad hoc algebraic manipulations. The aforementioned application of estimating the position of the human body in the sagittal plan based on accelerometer measurements [15] is also based on an algebraic technique from which the idea is a bit alike the one presented in this paper.

In Section III, we formalize our estimation problem. The algebraic framework for our method is described in Section IV. The results for small-dimensional cases, as well as for the general problem can be found in Section V. Numerical simulations are provided in Section VI to illustrate the efficiency of our algebraic method, comparing it with the Modified Prony method.

\section{NOTATIONS}

The vector containing all parameters involved in the signal is denoted by $\Theta$. It contains the subset $\Theta_{\text {est }}$ with the parameters to be estimated and $\Theta_{\overline{\mathrm{est}}}$ with the undesired ones.

We denote by $\mathbb{K}$ a field of characteristic zero and by $\mathbb{K}(s)\left[\frac{d}{d s}\right]$ the (non-commutative) polynomial ring in the differential operator $\frac{d}{d s}$ with coefficients in the field of fractions $\mathbb{K}(s)$. From $\mathbb{K}$ and a subset $\Upsilon \subset \Theta$, we build the algebraic extension $\mathbb{K}_{\Upsilon}:=\mathbb{K}(\Upsilon)$

The convolution operation is denoted by $\star$, that is, $f(t) \star g(t)=\int_{0}^{+\infty} f(t-\tau) g(\tau) d \tau$.

\section{PROBLEM FORMULATION}

Let us start with a signal depending on a set of parameters:

$$
x(t)=\sum_{k=1}^{n} \alpha_{k} \mathrm{e}^{i\left(\omega_{k} t+\phi_{k}\right)} .
$$

We wish to estimate amplitudes $\alpha_{k}$, frequencies $\omega_{k}$ and phases $\phi_{k}$ for all $k$. For that, we introduce parameters $\theta_{\ell}$ defined from $\alpha_{k}, \omega_{k}$ and $\phi_{k}$. Then, based on the observed noisy signal, our goal is to obtain a good approximation of these parameters $\theta_{\ell}$.

For $1 \leq \ell \leq n$, let us denote by $\theta_{\ell}$ a multiple of the elementary symmetric polynomial in $n$ variables $\omega_{1}, \ldots, \omega_{n}$ given by:

$$
\theta_{\ell}:=(-i)^{\ell} \sum_{1 \leq j_{1}<j_{2}<\cdots<j_{\ell} \leq n} \omega_{j_{1}} \omega_{j_{2}} \ldots \omega_{j_{\ell}} .
$$

So the $\theta_{k}$ can be obtained as the coefficients of the polynomial in the variable $X$ given by

$$
\prod_{\ell=1}^{n}\left(X-i \omega_{\ell}\right)=X^{n}+\theta_{1} X^{n-1}+\theta_{2} X^{n-2}+\cdots+\theta_{n} .
$$

The biased signal $z(t)=x(t)+\beta$ satisfies then a linear differential algebraic relation:

$$
z^{(n)}(t)+\sum_{\ell=1}^{n} \theta_{\ell} z^{(n-\ell)}(t)-\theta_{n} \beta=0 .
$$

We say that two set of parameters are equivalent if it is enough to determine one set in order to deduce the other. From the definition (3) of $\theta_{\ell}$ and relation (4), it is easy to prove:

Lemma 1: The sets of parameters $\left\{\omega_{1}, \ldots, \omega_{n}\right\}$ and $\left\{\theta_{1}, \ldots, \theta_{n}\right\}$ are equivalent.

For $1 \leq \ell \leq n$, let us set

$$
\theta_{n+\ell}:=-x^{(\ell-1)}(0)
$$

Moreover, the following Lemma holds (its proof can be found in Appendix A):

Lemma 2: Assume that the frequencies $\omega_{1}, \ldots, \omega_{n}$ are all known. Then the sets of parameters $\left\{\alpha_{1}, \phi_{1}, \ldots, \alpha_{n}, \phi_{n}\right\}$ and $\left\{\theta_{n+1}=-x(0), \theta_{n+2}=-\dot{x}(0), \ldots, \theta_{2 n}=-x^{(n-1)}(0)\right\}$ are equivalent.

Let us set $\theta_{2 n+1}:=-\beta$, the bias that we are not interested in estimating. Therefore, according to the above remarks, we want to estimate the set:

$$
\Theta:=\left\{\theta_{1}, \ldots, \theta_{n}, \theta_{n+1}, \ldots, \theta_{2 n}\right\} .
$$

We apply the Laplace transform on the equation (5) and obtain the following relation in the operational domain:

$$
s^{n} Z(s)-\sum_{j=0}^{n-1} z^{(n-1-j)}(0) s^{j}+\sum_{\ell=0}^{n-1} \theta_{n-\ell}\left(s^{\ell} Z(s)-\sum_{j=0}^{\ell-1} z^{(\ell-1-j)}(0) s^{j}\right)-\frac{\theta_{n} \beta}{s}=0 .
$$


Remark that $z(0)=x(0)+\beta=-\theta_{n+1}-\theta_{2 n+1}$ and $z^{(j)}(0)=x^{(j)}(0)=-\theta_{n+j+1}$, for $1 \leq j \leq n-1$. To simplify (7) and subsequent computations, we define the unitary polynomial in $\mathbb{C}_{\Theta}[s]$ :

$$
T_{\ell}(s):=s^{n-\ell}+\sum_{k=\ell}^{n-1} \theta_{n-k} s^{k-\ell} \text { for } 0 \leq \ell \leq n-1 \text { and } T_{n}(s)=1
$$

This polynomial has degree $n-\ell$ (in the variable $s$ ) and it satisfies the recurrence property:

$$
T_{\ell}(s)=s T_{\ell+1}(s)+\theta_{n-\ell}(0 \leq \ell \leq n-1) .
$$

So the equation $(7)$ reads

$$
s T_{0}(s) Z(s)+s \sum_{j=0}^{n-1} T_{j+1}(s) \theta_{n+j+1}+T_{0}(s) \theta_{2 n+1}=0 .
$$

Notice that $T_{0}(s)=s^{n}+\sum_{k=0}^{n-1} \theta_{n-k} s^{k}$ depends on the set $\left\{\theta_{1}, \ldots, \theta_{n}\right\}$. Similarly, $T_{\ell}(s)$ depends on $\left\{\theta_{1}, \ldots, \theta_{n-\ell}\right\}$, for any $1 \leq \ell \leq n$. So, regarding our estimation problem, we can set two goals:

Goal 1: frequencies estimation, i.e. identifying the parameters $\left\{\theta_{1}, \ldots, \theta_{n}\right\}$,

Goal 2: amplitudes and phases estimation, i.e identifying the parameters $\left\{\theta_{n+1}, \ldots, \theta_{2 n}\right\}$.

In this work, we propose solutions to these questions. Remark that this is equivalent to estimate some subset of parameters in $\Theta$, hence we shall use the notation $\Theta_{\text {est }}$ for the set of parameters to be estimated and $\Theta_{\overline{\text { est }}}$ for the set of undesired parameters (it will always contain the bias $\theta_{2 n+1}=-\beta$ ).

After eliminating $\Theta_{\overline{\mathrm{est}}}$ in the equation (9), we obtain a system of equations depending uniquely on $\Theta_{\text {est. }}$ Furthermore, one may distinguish two sub-cases for Goal 2: simultaneous and individual estimation of $\left\{\theta_{n+1}, \ldots, \theta_{2 n}\right\}$. Hence we may consider three cases:

Case 1: frequencies estimation: we set $\Theta_{\text {est }}=\left\{\theta_{1}, \ldots, \theta_{n}\right\}$ and $\Theta_{\overline{\mathrm{est}}}=\left\{\theta_{n+1}, \ldots, \theta_{2 n}, \theta_{2 n+1}\right\}$.

Case 2: simultaneous amplitudes and phases estimation: use the estimation of the frequencies and set $\Theta_{\text {est }}=\left\{\theta_{n+1}, \ldots, \theta_{2 n}\right\}$ and $\Theta_{\overline{\mathrm{est}}}=\left\{\theta_{2 n+1}\right\}$.

Case 3: individual amplitudes and phases estimation: use the estimation of the frequencies and start by setting $\Theta_{\text {est }}=\left\{\theta_{n+1}\right\}$ and $\Theta_{\overline{\mathrm{est}}}=\left\{\theta_{n+2}, \ldots, \theta_{2 n}, \theta_{2 n+1}\right\}$. Then use the estimation of $\theta_{n+1}$ to estimate $\theta_{n+2}$ and set $\Theta_{\text {est }}=\left\{\theta_{n+2}\right\}$ and $\Theta_{\overline{\text { est }}}=\left\{\theta_{n+3}, \ldots, \theta_{2 n}, \theta_{2 n+1}\right\}$. And so on, for each $1 \leq \ell \leq n, \Theta_{\text {est }}=\left\{\theta_{n+\ell}\right\}, \Theta_{\overline{\text { est }}}=\left\{\theta_{n+\ell+1}, \theta_{n+\ell+2}, \ldots, \theta_{2 n}, \theta_{2 n+1}\right\}$.

Now, let us consider the algebraic extensions $\mathbb{C}_{\Theta_{\mathrm{est}}}:=\mathbb{C}\left(\Theta_{\mathrm{est}}\right)$ and $\mathbb{C}_{\Theta}:=\mathbb{C}(\Theta)$ and the polynomial rings $\mathbb{C}_{\Theta_{\mathrm{est}}}[s]$ and $\mathbb{C}_{\Theta}[s]$. The relation below arises naturally from equation (9):

$$
\mathcal{R}\left(s, Z(s), \Theta_{\mathrm{est}}, \Theta_{\overline{\mathrm{est}}}\right):=P(s) Z(s)+Q(s)+\bar{Q}(s)=0,
$$

where $P(s)=s T_{0}(s), Q(s)$ is a polynomial in $s$ with coefficients only in the set of desired parameters $\Theta_{\text {est }}$ (i.e. they belong to $\left.\mathbb{C}_{\Theta_{\text {est }}}\right)$ and $\bar{Q}(s)$ contains the remaining terms. Hence $\bar{Q} \in \mathbb{C}_{\Theta}[s]$ is a linear combination of elements in $\Theta_{\overline{\text { est }}}$ with coefficients in $\mathbb{C}_{\Theta_{\mathrm{est}}}[s]$. For instance, let us examine the polynomials $Q(s)$ and $\bar{Q}(s)$ in the three cases mentioned above:

Case 1:

$$
\begin{aligned}
& Q(s)=0 \\
& \bar{Q}(s)=s \sum_{j=0}^{n-1} T_{j+1}(s) \theta_{n+j+1}+T_{0}(s) \theta_{2 n+1}
\end{aligned}
$$

Case 2:

$$
\begin{aligned}
& Q(s)=s \sum_{j=0}^{n-1} T_{j+1}(s) \theta_{n+j+1}, \\
& \bar{Q}(s)=T_{0}(s) \theta_{2 n+1}
\end{aligned}
$$

Case 3: for each $\ell \in\{0, \ldots, n-1\}$

$$
\begin{aligned}
& Q(s)=s \sum_{j=0}^{\ell} T_{j+1}(s) \theta_{n+j+1}, \\
& \bar{Q}(s)=s \sum_{j=\ell+1}^{n-1} T_{j+1}(s) \theta_{n+j+1}+T_{0}(s) \theta_{2 n+1}
\end{aligned}
$$

Notice that in all three cases, the degree in $s$ of the polynomial $\bar{Q}$ is $n$. As mentioned earlier, we start by eliminating the undesired parameters in $\Theta_{\overline{\text { est }}}$. In other words, we annihilate $\bar{Q}$ by applying some differential operators on the relation $\mathcal{R}$ (10). These operators will be written in a normal form, called the canonical form defined by the structural properties of the algebra 
underlying. Moreover, the differential operators form a principal ideal of the algebra, hence generated by a single operator called minimal $\bar{Q}$-annihilator.

To resume, the procedure can be described in three steps as enumerated below.

Procedure 1:

1) Algebraic elimination of $\Theta_{\overline{\text { est }}}$ : apply the minimal $\bar{Q}$-annihilator on the relation $\mathcal{R}$.

2) Obtaining a system of equations on $\Theta_{\text {est }}$ : apply the canonical form of differential operators generated by the minimal $\bar{Q}$-annihilator. This will provide a system of equations with good numerical properties in the time domain.

3) Resolution of the system: bring the equations back to the time domain by using the inverse Laplace transform

$$
\mathcal{L}^{-1}\left(\frac{1}{s^{m}} \frac{d^{p} Z(s)}{d s^{p}}\right)=\frac{(-1)^{p} t^{m+p}}{(m-1) !} \int_{0}^{1} w_{m-1, p}(\tau) z(t \tau) d \tau,
$$

with $w_{m, p}(\tau)=(1-\tau)^{m} \tau^{p}$, for all $p, m \in \mathbb{N}, m \geq 1$. We also use a shorter notation $w_{m, p}=w_{m, p}(\tau)$. To reduce the noise interference in the estimation, choose the integers $m$ and $p$ as small as possible. A more general following convolution result is:

$$
\mathcal{L}^{-1}\left(\frac{g(s)}{s^{m}} \frac{d^{p} Z(s)}{d s^{p}}\right)=g(t) \star W_{m, p}(t)
$$

with $\quad W_{m, p}(t)=\frac{(-1)^{p} t^{m+p}}{(m-1) !} \int_{0}^{1} w_{m-1, p}(\tau) \quad z(t \tau) d \tau, \quad$ for $\quad$ all $\quad p, \quad m \quad \in \mathbb{N}, \quad m \geq 1, \quad$ since $\mathcal{L}^{-1}\left(\frac{g(s)}{s^{m}} \frac{d^{p} Z(s)}{d s^{p}}\right)=\frac{(-1)^{p}}{(m-1) !} \int_{0}^{+\infty} g\left(t-\tau_{1}\right) \tau_{1}^{m+p} \int_{0}^{1} w_{m-1, p}\left(\tau_{2}\right) z\left(\tau_{1} \tau_{2}\right) d \tau_{2} d \tau_{1}$ where $\mathcal{L}^{-1}(g(s))=g(t)$.

In next section, we provide an overview of the algebraic formalism used to define our estimation method. We also define the minimal annihilators mentioned previously. The canonical form of the annihilators is defined in subsection IV-A.

\section{AnNihilators}

The inspiration for the algebraic framework comes from the work of M. Fliess et al. [47], [46], [50], [49], [52] ${ }^{1}$. The reader may find more details about the algebraic notions in [68] and [69].

Recall that our first goal is to annihilate the polynomial $\bar{Q} \in \mathbb{C}_{\Theta}[s]$ of degree $n$. For that, a natural idea is to use a differential operator in $\frac{d}{d s}$, meaning an operator of the form $\Pi=A_{0}+A_{1} \frac{d}{d s}+A_{2} \frac{d^{2}}{d s^{2}}+\cdots+A_{r} \frac{d^{r}}{d s^{r}}$ for some $r \in \mathbb{N}$ where the $A_{i}$ are elements of the field $\mathbb{K}\left(\mathbb{K}=\mathbb{C}\right.$ or $\left.\mathbb{C}_{\Theta}(s)\right)$. The positive integer $r$ is the order of the operator $\Pi$, i.e. its degree as a polynomial in the variable $\frac{d}{d s}$. It is easy to see that to eliminate $\bar{Q}$, it is enough to apply that operators with lowest degree in $\frac{d}{d s}$ strictly bigger than $n$. For example, $\Pi_{1}=\frac{d^{n+2}}{d s^{n+2}}-2 \frac{d^{n+1}}{d s^{n+1}}, \Pi_{2}=\left(s \frac{d}{d s}-n\right) \circ \cdots \circ\left(s \frac{d}{d s}-1\right) \circ\left(s \frac{d}{d s}\right)$ or $\Pi_{3}=\frac{d^{n+1}}{d s^{n+1}}$.

Hence, there are many choices for the sought differential operator. Intuitively, we can imagine that some possible operators coincide, even if they are written differently. For instance, do $\Pi_{2}$ and $\Pi_{3}$ above represent the same operator? In this case, the answer is positive, we refer to Corollary 1 . Another relevant question is whether an operator having order smaller than $n$ annihilates $\bar{Q}$. As we shall see later, that depends on the case we are examining: the answer is negative in Case 1 , but positive in Cases 2 and 3 (see previous Section). These answers are provided by the properties of the Weyl algebra structure of $\mathbb{C}_{\Theta}[s]\left[\frac{d}{d s}\right]$.

\section{A. Weyl Algebra}

Here we review some well-known properties of the Weyl algebra that are useful in the sequel. For more details and proofs of the Propositions, see for instance [69].

Let $k \in \mathbb{N}$. The Weyl algebra $\mathrm{A}_{k}(\mathbb{K})$ is the $\mathbb{K}$-algebra generated by $p_{1}, q_{1}, \ldots, p_{k}, q_{k}$ satisfying $\left[p_{i}, q_{j}\right]=\delta_{i j},\left[p_{i}, p_{j}\right]=$ $\left[q_{i}, q_{j}\right]=0, \forall 1 \leq i, j \leq k$ where $[\cdot, \cdot]$ is the commutator defined by $[u, v]:=u v-v u, \forall u, v \in \mathrm{A}_{k}(\mathbb{K})$. We will simply write $A_{k}$ instead of $A_{k}(\mathbb{K})$ when we do not need to make explicit the base field. A well-known fact is that $A_{k}$ can be realized as the algebra of polynomial differential operators on the polynomial ring in $k$ indeterminates $\mathbb{K}\left[s_{1}, \ldots, s_{k}\right]$ by setting $p_{i}=\frac{\partial}{\partial s_{i}}$ and $q_{i}=s_{i} \times \cdot, \forall 1 \leq i \leq k$. Using the same notation for the variable $s_{i}$ and for the operator multiplication by $s_{i}$, we have $\mathrm{A}_{k}=\mathbb{K}\left[q_{1}, \ldots, q_{k}\right]\left[p_{1}, \ldots, p_{k}\right]=\mathbb{K}\left[s_{1}, \ldots, s_{k}\right]\left[\frac{\partial}{\partial s_{1}}, \ldots, \frac{\partial}{\partial s_{k}}\right]$ A closely related algebra to $\mathrm{A}_{k}$ is formed by the differential operators on $\mathbb{K}\left[s_{1}, \ldots, s_{k}\right]$ with coefficients in the field of rational functions $\mathbb{K}\left(s_{1}, \ldots, s_{k}\right)$, denote it by $\mathrm{B}_{k}(\mathbb{K})=$ $\mathrm{B}_{k}:=\mathbb{K}\left(q_{1}, \ldots, q_{k}\right)\left[p_{1}, \ldots, p_{k}\right]=\mathbb{K}\left(s_{1}, \ldots, s_{k}\right)\left[\frac{\partial}{\partial s_{1}}, \ldots, \frac{\partial}{\partial s_{k}}\right] . \mathrm{A}_{k}$ is given by $\left\{q^{I} p^{J} \mid I, J \in \mathbb{N}^{k}\right\}$ where $q^{I}:=q_{1}^{i_{1}} \ldots q_{k}^{i_{k}}$ and $p^{J}:=p_{1}^{j_{1}} \ldots p_{k}^{j_{k}}$. Thus, any $F \in \mathrm{A}_{k}$ is written as $F=\sum_{I, J} \lambda_{I J} q^{I} p^{J}$, where $\lambda_{I J} \in \mathbb{K}$.

\footnotetext{
${ }^{1}$ Similar tools were also used for numerical differentiation of noisy signal [55], [58] and spike detection [67].
} 
Lemma 3: The following identities are valid:

$$
\begin{aligned}
& q^{m} p^{n}=p^{n} q^{m}+\sum_{k=1}^{m}\left(\begin{array}{l}
m \\
k
\end{array}\right)\left(\begin{array}{l}
n \\
k
\end{array}\right) k !(-1)^{k} p^{n-k} q^{m-k} \\
& p^{n} q^{m}=q^{m} p^{n}+\sum_{k=1}^{n}\left(\begin{array}{l}
n \\
k
\end{array}\right)\left(\begin{array}{l}
m \\
k
\end{array}\right) k ! q^{m-k} p^{n-k}
\end{aligned}
$$

Using the identities above, an induction proof shows that:

Corollary 1: For any $n \in \mathbb{N}$, one has $\left(s \frac{d}{d s}-n\right) \circ \cdots \circ\left(s \frac{d}{d s}-1\right) \circ\left(s \frac{d}{d s}\right)=s^{n+1} \frac{d^{n+1}}{d s^{n+1}}$.

Corollary 2: For any $m \in \mathbb{N}$, one has: $\left(s \frac{d}{d s}-1\right) \circ \cdots \circ\left(s \frac{d}{d s}-m\right)=\sum_{j=0}^{m} \sum_{k=j}^{m} \mathbf{s}(m+1, k+1) \mathbf{S}(k, j) s^{j} \frac{d^{j}}{d s^{j}}$ where $\mathbf{s}(m, k)$ are the Stirling numbers of the first kind with generating function $\sum_{k=0}^{m} \mathbf{S}(m, k) x^{k}=(x)_{m}$ and $\mathbf{S}(k, j)$ are the Stirling numbers of the second kind with generating function $\sum_{j=0}^{k} \mathbf{S}(k, j)(x)_{j}=x^{k}$ with $(x)_{m}=x(x-1) \ldots(x-m+1)$ the falling factorial of $x$.

Proof: Recall that the Euler operator $\mathrm{E}=s \frac{d}{d s}$ commutes with itself, therefore $(\mathrm{E}-1) \circ \cdots \circ(\mathrm{E}-m)=\sum_{k=0}^{m} \mathbf{s}(m+1, k+1) \mathrm{E}^{k}$. Since $\mathrm{E}^{k}=\sum_{j=0}^{k} \mathrm{~S}(k, j) \frac{d^{j}}{d s^{j}}$, then the result follows.

Similarly to elements in $\mathrm{A}_{k}$, we define:

Definition 1: Let $F \in \mathrm{B}_{k}$. We say that $F$ is in its canonical form if $F=\sum_{I \in \mathbb{N}^{k}} g_{I}(q) p^{I} \quad$ where $g_{I}(q) \in \mathbb{K}\left(q_{1}, \ldots, q_{k}\right)$.

The order of an element $F=\sum_{\text {finite }} g_{I}(q) p^{I} \in \mathrm{B}_{k}$ is defined as $\operatorname{ord}(F):=\max \left\{\left[I|| g_{I}(q) \neq 0\right\}\right.$ with $|I|:=i_{1}+\cdots+i_{k}$ if $I=\left(i_{1}, \ldots, i_{k}\right) \in \mathbb{N}^{k}$. An immediate consequence of this definition is $\operatorname{ord}(F G)=\operatorname{ord}(F)+\operatorname{ord}(G)$, for all $F$ and $G \in \mathrm{B}_{k}$.

There are no left or right zero divisors neither in $A_{k}$, nor in $B_{k}$, then $B_{k}$ is a domain and so is $A_{k}$. Moreover, $A_{k}$ and $B_{k}$ are simple and Noetherian. In the case $k=1$, a very important property holds:

Proposition 1: B admits a left division algorithm, that is, if $F, G \in \mathrm{B}$, then there exists $Q, R \in \mathrm{B}_{1}$ such that $F=Q G+R$ and $\operatorname{ord}(R)<\operatorname{ord}(G)$. As a consequence, B is a principal left domain.

However, remark that $A_{k}$ is neither a principal right domain, nor a principal left domain. Finally, since $\frac{d}{d s}$ is a derivation operator, we have a useful property:

Proposition 2 (Derivation): Let $F, G \in \mathbb{K}[s]$. We have $\frac{d^{n}}{d s^{n}}(F G)=\sum_{k=0}^{n}\left(\begin{array}{l}n \\ k\end{array}\right) \frac{d^{k} F}{d s^{k}} \frac{d^{n-k} G}{d s^{n-k}}$ (Leibniz rule).

\section{B. Annihilator}

Definition 2: Let $R \in \mathbb{C}_{\Theta}[s]$ and $\mathrm{B}=\mathbb{C}(s)\left[\frac{d}{d s}\right]$. Consider $\operatorname{Ann}_{\mathrm{B}}(R)=\{F \in \mathrm{B} \mid F(R)=0\}$. An element of the left ideal $\operatorname{Ann}_{\mathrm{B}}(R)$ is called a $R$-annihilator with respect to $\mathrm{B}$.

Remark 1: From Proposition 1, it follows that $\operatorname{Ann}_{\mathrm{B}}(R)$ is a left principal ideal. So it is generated by a single generator $\Pi_{\text {min }} \in \mathrm{B}$ called a minimal $R$-annihilator (with respect to $\mathrm{B}$ ). We have $\operatorname{Ann}_{\mathrm{B}}(R)=\mathrm{B} \Pi_{\text {min. }}$. The generator $\Pi_{\text {min }}$ is unique up to multiplication by an operator in $\mathrm{B}$. We notice that $\operatorname{Ann}_{\mathrm{B}}(R)$ contains annihilators in finite integral form, i.e. operators with coefficients in $\mathbb{C}\left[\frac{1}{s}\right]$.

Remark 2: Remark 1 still holds if the field $\mathbb{C}$ is replaced by $\mathbb{C}_{\Upsilon}$ in the Definition 2 , with $\Upsilon \subseteq \Theta$. We write $\mathrm{B}_{\Upsilon}:=\mathbb{C}_{\Upsilon}(s)\left[\frac{d}{d s}\right]$. Hence $\operatorname{Ann}_{\mathrm{B}_{\Upsilon}}(R)$ is generated by a unique generator in $\mathrm{B}_{\Upsilon}$ (up to multiplication by a polynomial in $\mathbb{C}_{\Upsilon}(s)$ ) called a minimal $R$-annihilator w.r.t. $\mathrm{B}$ r.

Lemma 4: Consider $Q_{n}(s)=s^{n}, n \in \mathbb{N}$. A minimal $Q_{n}$-annihilator with respect to B is $\Pi_{n}=s \frac{d}{d s}-n$.

Proof: It is clear that $\Pi_{n}\left(Q_{n}\right)=0$. Moreover, if $\Pi$ is a generator of $\operatorname{Ann}_{\mathrm{B}}\left(Q_{n}\right)$, then $\Pi_{n}=F$. $\Pi$ with $F \in \mathrm{B}$. But ord $\left(\Pi_{n}\right)=1$, so $\Pi$ must have order equal to 1 . Hence $\Pi_{n}$ is also a generator, hence a minimal $Q_{n}$-annihilator.

Clearly, this annihilator is unique up to a multiplication by a polynomial in $\mathbb{C}(s)$. Let us note that for $m, n \in \mathbb{N}$, the operators $\Pi_{m}$ and $\Pi_{n}$ commute. The following lemmas are useful:

Lemma 5: Let $P_{1}, P_{2} \in \mathbb{C}_{\Theta}[s]$. Let $\Pi_{1}$ be a $P_{1}$-annihilator and $\Pi_{2}$ be a $P_{2}$-annihilator such that $\Pi_{1} \Pi_{2}=\Pi_{2} \Pi_{1}$. Then $\Pi_{1} \Pi_{2}$ is a $\left(\mu P_{1}+\eta P_{2}\right)$-annihilator for all $\mu, \eta \in \mathbb{C}_{\Theta}$.

Corollary 3: Consider $\bar{Q}(s)=s \sum_{j=0}^{n-1} T_{j+1}(s) \theta_{n+1+j}+T_{0}(s) \theta_{2 n+1} \in \mathbb{C}_{\Theta}[s]$ (see eq. (12)). Then a minimal $\bar{Q}$-annihilator w.r.t. $\mathrm{B}$ is $\Pi_{\min }=s^{n+1} \frac{d^{n+1}}{d s^{n+1}}$.

Proof: The degree of $\bar{Q}$ in the variable $s$ is $n$, so it is clear that $\Pi_{\min }$ annihilates $\bar{Q}$. Assume that $\Pi \in \mathrm{B}$ is a generator of $\operatorname{Ann}_{\mathrm{B}}(\bar{Q})$. Since $\Pi$ annihilates $\bar{Q}$, it must have order greater or equal to $n+1$. We can write $\Pi_{\text {min }}=F \Pi$ for some $F \in \mathrm{B}$, then comparing orders, we obtain ord $(\Pi)=n+1$ and $\operatorname{ord}(F)=0$. Moreover, writing both operators in the canonical form, it results that $F=1$ and $\Pi_{\min }=\Pi$. 
Lemma 6: Let $\widetilde{\Theta} \subset \Theta$ be a set consisting of some (already) estimated parameters of $\Theta$. Let $R \in \mathbb{C}_{\widetilde{\Theta}}[s]$. Then a minimal $R$-annihilator w.r.t $\mathrm{B}_{\widetilde{\Theta}}$ is $\Pi_{\min }=R \frac{d}{d s}-\frac{d R}{d s}$.

Proof: This proof is completely similar to the previous one. It is obvious that $\Pi_{\min }$ annihilates $R$. Assume that $\Pi$ is a generator of $\operatorname{Ann}_{\mathrm{B}_{\widetilde{\Theta}}}(R)$. We can write $\Pi_{\text {min }}=F \Pi$ for some $F \in \mathrm{B}_{\widetilde{\Theta}}$. Comparing orders on both sides, we obtain ord $(\Pi)=1$ and $\operatorname{ord} F)=0$. Furthermore, writing both operators in the canonical form, it results that $F=1$ and $\Pi_{\min }=\Pi$.

Corollary 4: Let $\ell \in\{0, \ldots, n-1\}$. Assume that the parameters in the set $\widetilde{\Theta}=\left\{\theta_{1}, \ldots, \theta_{n+\ell}\right\}$ are estimated. Denote by $\Pi_{\ell}=\left(s \frac{d}{d s}-(n-\ell-1)\right) \circ \cdots \circ\left(s \frac{d}{d s}-1\right)$. A minimal annihilator for $\bar{Q}=s \sum_{j=\ell+1}^{n-1} T_{j+1}(s) \theta_{n+j+1}+T_{0}(s) \theta_{2 n+1}$ w.r.t $\mathrm{B}_{\widetilde{\Theta}}$ is $\Pi_{\min }=\left(\Pi_{\ell}\left(T_{0}\right) \frac{d}{d s}-\frac{d \Pi_{\ell}\left(T_{0}\right)}{d s}\right) \circ \Pi_{\ell}$

Proof: By Corollary 2, we have $\Pi_{\ell}=\left(s \frac{d}{d s}-(n-\ell-1)\right) \circ \cdots \circ\left(s \frac{d}{d s}-1\right)=\sum_{k=0}^{n-\ell-1} \mathbf{s}(n-\ell, k+1) s^{k} \frac{d^{k}}{d s^{k}}$. This operator has order $n-\ell-1$, so its action on each $T_{j+1}$ for $\ell+1 \leq j \leq n-1$ is zero, since $\operatorname{deg}_{s}\left(T_{j+1}\right)=n-(j+1)$. So $\Pi_{\ell}(\bar{Q})=\Pi_{\ell}\left(T_{0}\right)$ and it follows easily that $\Pi_{\min }(\bar{Q})=0$. Remark that ord $\left(\Pi_{\min }\right)=n-\ell$.

To prove that $\Pi_{\min }$ is indeed a minimal annihilator, we begin with the observation that there are $n-\ell$ coefficients to be eliminated, corresponding to the coefficients of $\theta_{n+\ell+2}, \ldots, \theta_{2 n}, \theta_{2 n+1}$. Namely, they are respectively the polynomials $T_{\ell+2}$, $T_{\ell+3}, \ldots, T_{n}$ and $T_{0} \in \mathbb{C}_{\widetilde{\Theta}}[s]$. Apart from the last polynomial, $T_{\ell+2}$ is the polynomial with highest degree $n-\ell-2$. So, to annihilate $T_{\ell+2}, T_{\ell+3}, \ldots, T_{n}$, we must have an operator of order at least $n-\ell-1$. Using the Lemma above, to annihilate $T_{0}$ of degree $n$, we may complete it with an order 1 operator. Hence, the minimal annihilator must have order bigger than $n-\ell$.

\section{PARAMETER ESTIMATION}

As we have seen in Section III, we have the relation below (10) in the operational domain:

$$
\mathcal{R}\left(s, Z(s), \Theta_{\mathrm{est}}, \Theta_{\overline{\mathrm{est}}}\right)=P(s) Z(s)+Q(s)+\bar{Q}(s)=0 .
$$

According to Procedure 1, the first step of the estimation process is to annihilate the polynomial $\bar{Q}$. For that, we use the notion of a minimal annihilator (see Section IV), denote it by $\Pi_{\mathrm{min}}$.

The second step of the procedure is to determine a system of equations. For that, we choose a suitable family of annihilators $\mathscr{F}=\left(\Pi_{i}\right)_{i=1}^{r}$ in $\mathbb{C}_{\Theta}(s)\left[\frac{d}{d s}\right]$ generated by $\Pi_{\min }$ so that $\mathscr{F}$ applied to the relation above $\mathcal{R}$ provides the sought equations in $\Theta_{\text {est }}$.

Finally in the third step, the Laplace inverse transform applied to the solutions of this system provides the estimation of the desired parameters $\Theta_{\text {est }}$.

The order of the differential operators is one of the factors that must be taken in account when choosing the family $\mathscr{F}$ : it should be minimal to reduce noise sensitivity. Also, the use of finite-integral form annihilators is justified by (17) in the third step. In addition, the choice of a well-balanced system of equations implies good numerical properties.

In what follows, there are three subsections. We begin with the simplest case: one sinusoidal waveform signal. This simple example justifies first, why annihilators in two different sets B (annihilator with real coefficients) and $B_{\Theta}$ (annihilator with $\Theta$-dependent coefficients) are needed. Secondly, this one-dimensional case shows how our method is really efficient. Then, we present the case $n=2$ where two different solutions are given to this estimation problem. Clearly this second example shows how the complexity of the estimation problem grows with $n$ and gives hints to provide a useful solution in the general case. At last, the general case is presented. As mentioned in the Introduction, the algebraic approach considered in this work allows the proposition of original closed formulas for the parameter estimation. The differential algebra framework settled in the previous sections is used to develop new explicit expressions for the estimates.

\section{A. A single sinusoidal waveform signal}

In the case $n=1$, the signal is given by $x(t)=\alpha \mathrm{e}^{i(\omega t+\phi)}$. Using the notation in Section III, we have $\theta_{1}=-i \omega, \theta_{2}=-x(0)=$ $\beta-z(0)$ and $\theta_{3}=-\beta$. Thus the biased signal $z(t)=x(t)+\beta$ satisfies the differential equation $z^{\prime}(t)+\theta_{1} z(t)+\theta_{1} \theta_{3}=0$. In the operational domain, this expression reads as $s\left(s+\theta_{1}\right) Z(s)+s \theta_{2}+\left(s+\theta_{1}\right) \theta_{3}=0$. Setting $T_{0}(s)=s+\theta_{1}$ and $T_{1}(s)=1$, that provides:

$$
s T_{0}(s) Z(s)+s T_{1}(s) \theta_{2}+T_{0}(s) \theta_{3}=0 .
$$

1) Frequency Estimation: The frequency estimation corresponds to Case 1 in Section III. Estimating the frequency $\omega$ is equivalent to estimate $\theta_{1}$, as remarked in Lemma 1 . We set $\Theta_{\text {est }}=\left\{\theta_{1}\right\}$ and $\Theta_{\overline{\mathrm{est}}}=\left\{\theta_{2}, \theta_{3}\right\}$. Following equations (11) and (12), the polynomials $P, Q$ and $\bar{Q}$ in (10) are given by

$$
P(s)=s T_{0}(s), \quad Q(s)=0 \quad \text { and } \quad \bar{Q}(s)=s T_{1}(s) \theta_{2}+T_{0}(s) \theta_{3} .
$$


Using Corollary 3, a minimal $\bar{Q}$-annihilator w.r.t. B is:

$$
\Pi_{\min }=s^{2} \frac{d^{2}}{d s^{2}}
$$

Since $\frac{d^{2}}{d s^{2}}(P(s) Z(s))=P(s) \frac{d^{2} Z(s)}{d s^{2}}+2 P^{\prime}(s) \frac{d Z(s)}{d s}+\frac{d^{2} P(s)}{d s^{2}} Z(s)$ by Proposition 2, then

$$
\Pi_{\min }(P(s) Z(s))=s^{2}\left(p_{2}(s) \frac{d^{2} Z(s)}{d s^{2}}+p_{1}(s) \frac{d Z(s)}{d s}+p_{0}(s) Z(s)\right)
$$

where $p_{2}(s)=s\left(s+\theta_{1}\right), p_{1}(s)=2\left(2 s+\theta_{1}\right)$ and $p_{0}(s)=2$. Moreover $\Pi_{\min }(Q(s))=\Pi_{\min }(\bar{Q}(s))=0$. Thus, applying $\Pi_{\min }$ on relation (10) gives the following algebraic relation

$$
p_{2}(s) \frac{d^{2} Z(s)}{d s^{2}}+p_{1}(s) \frac{d Z(s)}{d s}+p_{0}(s) Z(s)=0
$$

leading to

$$
A(s) \theta_{1}=-B(s)
$$

with $A(s)=s^{3} \frac{d^{2} Z(s)}{d s^{2}}+2 s^{2} \frac{d Z(s)}{d s}$ and $B(s)=s^{4} \frac{d^{2} Z(s)}{d s^{2}}+4 s^{3} \frac{d Z(s)}{d s}+2 s^{2} Z(s)$. In order to apply (17) and to obtain this equation in the time domain, we have to divide the whole expression by an appropriate power of $s$, in this case a power greater than 5 . The resulting expression for $\theta_{1}$ after dividing (21) by $s^{5}$ and applying the Laplace inverse transform is:

$$
\theta_{1}=-\frac{1}{t} \frac{a(t)}{b(t)} \text { with } a(t)=\int_{0}^{1}\left(-4 w_{1,1}+w_{0,2}+w_{2,0}\right) z(t \tau) d \tau \text { and } b(t)=\int_{0}^{1}\left(-w_{2,1}+w_{1,2}\right) z(t \tau) d \tau
$$

An expression using the convolution product can also be obtained $\theta_{1}=-\frac{(g \star a)(t)}{(g \star b)(t)}$.

2) Amplitude and phase estimation: The estimation of $\theta_{2}$ is equivalent to the estimation of the amplitude $\alpha$ and the phase $\phi$. We repeat the algorithm seen in the previous subsection. An important remark is that the estimation of $\theta_{1}$ obtained above can be used in the sequel. We set $\Theta_{\text {est }}=\left\{\theta_{2}\right\}$ and $\Theta_{\text {est }}=\left\{\theta_{3}\right\}$. Following equations (15) and (16), the polynomials $P, Q$ and $\bar{Q}$ in (10) are given by $P(s)=s T_{0}(s), Q(s)=s T_{1}(s) \theta_{2}$ and $\bar{Q}=T_{0}(s) \theta_{3}$. Recall that to estimate the frequency, we used a minimal $\bar{Q}$-annihilator w.r.t. B given by (20), allowing us to linearly identify the parameter $\theta_{1}$. A nonlinear equation on $\theta_{2}$ is found by using the annihilator that depends on $\theta_{1}$, that means we can look for an annihilator in $\mathrm{B}_{\Theta}$. From Corollary 4 , since $T_{0}(s)=s+\theta_{1}$, we obtain the $\bar{Q}$-annihilator w.r.t. $\mathrm{B}_{\Theta}$ :

$$
\Pi_{\min }^{\Theta}=T_{0}(s) \frac{d}{d s}-1=\left(s+\theta_{1}\right) \frac{d}{d s}-1 .
$$

Since $\frac{d}{d s}(P(s) Z(s))=\frac{d P(s)}{d s} Z(s)+P(s) \frac{d Z(s)}{d s}$ by Proposition 2, then applying the minimal annihilator on relation (19) gives $\Pi_{\min }^{\Theta}(P(s) Z(s))=T_{0}(s)^{2}\left(s \frac{d Z(s)}{d s}+Z(s)\right), \Pi_{\min }^{\Theta}(Q)=\theta_{1} \theta_{2}, \Pi_{\min }^{\Theta}(\bar{Q})=0$. The expression for $\theta_{2}$ is thus obtained:

$$
\theta_{2}=\theta_{1}^{2} t^{2} \frac{\int_{0}^{1}\left(w_{0,1}(\tau)-w_{1,0}(\tau)\right) z(t \tau) d \tau}{2 \exp \left(-\frac{\theta_{1} t}{2}\right)\left(\theta_{1} t \cosh \left(\frac{\theta_{1} t}{2}\right)-2 \sinh \left(\frac{\theta_{1} t}{2}\right)\right)} .
$$

Notice that we can also use a convolution with any function $g$.

\section{B. A sum of two sinusoidal waveform signals}

Let us now consider the sum of two sinusoidal waveform signals. The signal $x(t)$ is then $x(t)=\alpha_{1} \mathrm{e}^{i\left(\omega_{1} t+\phi_{1}\right)}+\alpha_{2} \mathrm{e}^{i\left(\omega_{2} t+\phi_{2}\right)}$. We use again the notation in Section III and set $\theta_{1}=-i\left(\omega_{1}+\omega_{2}\right), \theta_{2}=-\omega_{1} \omega_{2}, \theta_{3}=-x(0)=\beta-z(0), \theta_{4}=-\dot{z}(0)=-\dot{x}(0)$, $\theta_{5}=-\beta$. Among the unknown parameters, we wish to estimate $\theta_{1}, \theta_{2}, \theta_{3}$ and $\theta_{4}$ using the measured signal $y(t)$, but not the bias $\theta_{5}$. The biased signal $z(t)=x(t)+\beta$ satisfies the following differential equation $z^{\prime \prime}(t)+\theta_{1} z^{\prime}(t)+\theta_{2} z(t)+\theta_{2} \theta_{5}=0$. In the operational domain, this differential equation reads as:

$$
s\left(s^{2}+\theta_{1} s+\theta_{2}\right) Z(s)+s\left(s+\theta_{1}\right) \theta_{3}+s \theta_{4}+\left(s^{2}+\theta_{1} s+\theta_{2}\right) \theta_{5}=0,
$$

Using the polynomials $T_{i}$ defined in (8), we obtain:

$$
s T_{0}(s) Z(s)+s\left(T_{1}(s) \theta_{3}+T_{2}(s) \theta_{4}\right)+T_{0}(s) \theta_{5}=0 .
$$

with $T_{0}(s)=s^{2}+\theta_{1} s+\theta_{2}, T_{1}(s)=s+\theta_{1}$ and $T_{2}(s)=1$. 
1) Frequencies estimation: We begin with the estimation of $\theta_{1}$ and $\theta_{2}$. This is equivalent to frequencies estimation (see Lemma 1). So, we have two sets $\Theta_{\text {est }}=\left\{\theta_{1}, \theta_{2}\right\}$ and $\Theta_{\overline{\mathrm{est}}}=\left\{\theta_{3}, \theta_{4}, \theta_{5}\right\}$. According to equations (11) and (12), the polynomials $P, Q$ and $\bar{Q}$ in relation $\mathcal{R}(23)$ are $P(s)=s\left(s^{2}+\theta_{1} s+\theta_{2}\right), Q(s)=0$ and $\bar{Q}(s)=s\left(s+\theta_{1}\right) \theta_{3}+s \theta_{4}+\left(s^{2}+\theta_{1} s+\theta_{2}\right) \theta_{5}$. From Corollary 3, we find a minimal $\bar{Q}$-annihilator w.r.t. B given by $\Pi_{\min }=s^{3} \frac{d^{3}}{d s^{3}}$. Using Proposition 2 , we obtain

$$
\Pi_{\min }(P(s) Z(s))=s^{3}\left(p_{3}(s) \frac{d^{3} Z(s)}{d s^{3}}+p_{2}(s) \frac{d^{2} Z(s)}{d s^{2}}+p_{1}(s) \frac{d Z(s)}{d s}+p_{0}(s) Z(s)\right),
$$

where $p_{3}(s)=s\left(s^{2}+s \theta_{1}+\theta_{2}\right), p_{2}(s)=3\left(3 s^{2}+2 s \theta_{1}+\theta_{2}\right), p_{1}(s)=6\left(3 s+\theta_{1}\right), p_{0}(s)=6 . \operatorname{Moreover} \Pi_{\min }(Q(s))=\Pi_{\min }(\bar{Q}(s))=$ 0 . Thus applying $\Pi_{\min }$ on relation $\mathcal{R}(23)$ gives a single equation in $\theta_{1}$ and $\theta_{2}$ :

$$
A_{1}(s) \theta_{1}+A_{2}(s) \theta_{2}=-B(s)
$$

with $A_{1}(s)=6 s^{3} \frac{d Z(s)}{d s}+6 s^{4} \frac{d^{2} Z(s)}{d s^{2}}+s^{5} \frac{d^{3} Z(s)}{d s^{3}}, A_{2}(s)=3 s^{3} \frac{d^{2} Z(s)}{d s^{2}}+s^{4} \frac{d^{3} Z(s)}{d s^{3}}$ and $B(s)=6 s^{3} Z(s)+18 s^{4} \frac{d Z(s)}{d s}+9 s^{5} \frac{d^{2} Z(s)}{d s^{2}}+s^{6} \frac{d^{3} Z(s)}{d s^{3}}$.

To linearly identify these two parameters $\theta_{1}$ and $\theta_{2}$, we need two independent equations. However, we show in the Appendix $\mathrm{B}$, that this is not possible in the operational domain. Therefore, we shall use a construction in the time domain in two different ways:

Solution (A): Return to the time domain and convolute the result with two different functions.

Solution (B): Use $\bar{Q}$-annihilators leading to two independent equations in the time domain.

Let us detail these two solutions:

(A) To apply the inverse Laplace transform (17), we divide the expression (24) by $s^{7}$ and obtain $a_{1}(t) \theta_{1}+a_{2}(t) \theta_{2}=-b(t)$ with

$$
\begin{array}{r}
a_{1}(t)=t \int_{0}^{1}\left(-w_{1,3}+3 w_{2,2}-w_{3,1}\right)(\tau) \quad z(t \tau) \quad d \tau, \quad a_{2}(t)=\frac{t^{2}}{2} \int_{0}^{1}\left(w_{3},\right. \\
b(t)=\int_{0}^{1}\left(w_{3,0}-9 w_{2,1}+9 w_{1,2}-w_{0,3}\right)(\tau) z(t \tau) d \tau . \text { For arbitrary two functions } g_{1}(t) \\
\left(g_{1} \star a_{1}\right)(t) \theta_{1}+\left(g_{1} \star a_{2}\right)(t) \theta_{2}=-\left(g_{1} \star b\right)(t)
\end{array}
$$

That implies:

$$
\begin{aligned}
\theta_{1} & =-\frac{\left(g_{1} \star b\right)(t) \cdot\left(g_{2} \star a_{2}\right)(t)-\left(g_{1} \star a_{2}\right)(t) \cdot\left(g_{2} \star b\right)(t)}{\left(g_{1} \star a_{1}\right)(t) \cdot\left(g_{2} \star a_{2}\right)(t)-\left(g_{1} \star a_{2}\right)(t) \cdot\left(g_{2} \star a_{1}\right)(t)} \\
\theta_{2} & =-\frac{\left(g_{1} \star a_{1}\right)(t) \cdot\left(g_{2} \star b\right)(t)-\left(g_{2} \star a_{1}\right)(t) \cdot\left(g_{1} \star b\right)(t)}{\left(g_{1} \star a_{1}\right)(t) \cdot\left(g_{2} \star a_{2}\right)(t)-\left(g_{1} \star a_{2}\right)(t) \cdot\left(g_{2} \star a_{1}\right)(t)}
\end{aligned}
$$

(B) We have seen that $\bar{Q}$-annihilators are generated by the minimal annihilator $\Pi_{\min }=s^{3} \frac{d^{3}}{d s^{3}}$ (see Remark 1), so they are of the form $F \Pi_{\min }$ with $F \in \mathrm{B}=\mathbb{C}(s)\left[\frac{d}{d s}\right]$. To obtain two independent equations, we set $F=f_{0}(s)+f_{1}(s) \frac{d}{d s}$ with $f_{0}(s)$, $f_{1}(s) \in \mathbb{C}(s)$. Multiplying $\Pi_{\min }$ by $F$ on the left results in the $4^{\text {th }}$-order annihilator written in the canonical form:

$$
\Pi=g_{0}(s) \frac{d^{3}}{d s^{3}}+g_{1}(s) \frac{d^{4}}{d s^{4}},
$$

where $g_{0}(s)=s^{2}\left(f_{0}(s) s+3 f_{1}(s)\right), g_{1}(s)=s^{3} f_{1}(s) \in \mathbb{C}(s)$. The choice of $g_{0}(s)=1, g_{1}(s)=0$ and then $g_{0}(s)=0, g_{1}(s)=1$ provides two equations in the operational domain leading to the following system in the time domain:

$$
\left(\begin{array}{cccc}
t & I_{1} & \frac{t^{2}}{2} & I_{2} \\
t^{2} & I_{3} & \frac{t^{3}}{6} & I_{4}
\end{array}\right)\left(\begin{array}{c}
\theta_{1} \\
\theta_{2}
\end{array}\right)=\left(\begin{array}{c}
I_{5} \\
t \\
I_{6}
\end{array}\right)
$$

where

$$
\begin{aligned}
& I_{1}=\int_{0}^{1}\left(-w_{3,1}+3 w_{2,2}-w_{1,3}\right)(\tau) z(t \tau) d \tau, \quad I_{2}=\int_{0}^{1}\left(w_{3,2}-w_{2,3}\right)(\tau) z(t \tau) d \tau \\
& I_{3}=\int_{0}^{1}\left(2 w_{3,2}-4 w_{2,3}+w_{1,4}\right)(\tau) z(t \tau) d \tau, \quad I_{4}=\int_{0}^{1}\left(-4 w_{3,3}+3 w_{2,4}\right)(\tau) z(t \tau) d \tau \\
& I_{5}=\int_{0}^{1}\left(9 w_{2,1}-9 w_{1,2}+w_{0,3}-w_{3,0}\right)(\tau) z(t \tau) d \tau, \\
& I_{6}=\int_{0}^{1}\left(-18 w_{2,2}+12 w_{1,3}+4 w_{3,1}-w_{0,4}\right)(\tau) z(t \tau) d \tau
\end{aligned}
$$

Cramer's rule can be used to solve the above system and we find the expressions below:

$$
\theta_{1}=\frac{I_{4} I_{5}+3 I_{2} I_{6}}{t\left(I_{1} I_{4}-3 I_{2} I_{3}\right)} \quad \text { and } \quad \theta_{2}=\frac{6}{t^{2}} \frac{I_{1} I_{6}+I_{3} I_{5}}{\left(I_{1} I_{4}-3 I_{2} I_{3}\right)}
$$


2) Amplitudes and phases estimation: The estimation of amplitudes $\alpha_{1}, \alpha_{2}$ and phases $\phi_{1}$, $\phi_{2}$ is performed through the estimation of $\theta_{3}$ and $\theta_{4}$ as we claimed in Lemma 1 . We proceed by steps, first estimating $\theta_{3}$ and then, $\theta_{4}$. Hence, we first set $\Theta_{\text {est }}=\left\{\theta_{3}\right\}$ and $\Theta_{\overline{\text { est }}}=\left\{\theta_{4}, \theta_{5}\right\}$. An important remark is that the estimated values for $\theta_{1}$ and $\theta_{2}$ can be used in the estimation of $\theta_{3}$. For these sets $\Theta_{\text {est }}$ and $\Theta_{\overline{\text { est }}}$, the polynomials $P, Q$ and $\bar{Q}$ in relation $\mathcal{R}$ (see 10) are $P(s)=s T_{0}(s), Q(s)=s T_{1}(s) \theta_{3}$ and $\bar{Q}(s)=s T_{2}(s) \theta_{4}+T_{0}(s) \theta_{5}$ with $T_{0}(s)=s^{2}+\theta_{1} s+\theta_{2}, T_{1}(s)=s+\theta_{1}$ and $T_{2}(s)=1$. Using annihilators generated by the minimal $\bar{Q}$-annihilator w.r.t. B $:=\mathbb{C}(s)\left[\frac{d}{d s}\right]$ given by (25), we could linearly identify $\theta_{1}$ and $\theta_{2}$. From Theorem 1 , we know that it is not possible to identify linearly $\theta_{3}$ and $\theta_{4}$, so we will use nonlinear equations in $\theta_{1}$ and $\theta_{2}$. Corollary 4 indicates that a minimal $\bar{Q}$-annihilator w.r.t. $\mathrm{B}_{\Theta}$ is given by:

$$
\Pi_{\min }^{\Theta}=\left(\left(T_{0}(s)-s \frac{d T_{0}(s)}{d s}\right) \frac{d}{d s}+s \frac{d^{2} T_{0}(s)}{d s^{2}}\right) \circ\left(s \frac{d}{d s}-1\right) \frac{d}{d s}-s=2 s-2 s^{2} \frac{d}{d s}+s\left(s^{2}-\theta_{2}\right) \frac{d^{2}}{d s^{2}}
$$

written in the canonical form. We have:

$$
\begin{aligned}
\Pi_{\min }^{\Theta}(P(s) Z(s)) & =2 s\left(s^{3}-3 \theta_{2} s-\theta_{1} \theta_{2}\right) Z(s)+2 s\left(2 s^{4}+\theta_{1} s^{3}-3 \theta_{2} s^{2}-2 \theta_{1} \theta_{2} s-\theta_{2}^{2}\right) \frac{d Z(s)}{d s} \\
& +s^{2}\left(s^{4}+\theta_{1} s^{3}-\theta_{1} \theta_{2} s-\theta_{2}^{2}\right) \frac{d^{2} Z(s)}{d s^{2}} \\
\Pi_{\min }^{\Theta}(Q(s)) & =-s \frac{d^{2} T_{0}(s)}{d s^{2}} \theta_{2} \theta_{3}=-2 s \theta_{2} \theta_{3} \quad \text { and } \quad \Pi_{\min }^{\Theta}(\bar{Q}(s))=0
\end{aligned}
$$

where $T_{0}(s)=s^{2}+\theta_{1} s+\theta_{2} \in \mathbb{C}_{\Theta}[s]$. So, $\Pi_{\text {min }}^{\Theta_{\text {est }}}$ applied on $\mathcal{R}(10)$ gives the algebraic relation:

$$
\begin{array}{r}
2\left(s^{3}-3 \theta_{2} s-\theta_{1} \theta_{2}\right) Z(s)+2\left(2 s^{4}+\theta_{1} s^{3}-3 \theta_{2} s^{2}-2 \theta_{1} \theta_{2} s-\theta_{2}^{2}\right) \frac{d Z(s)}{d s}+s\left(s^{4}+\theta_{1} s^{3}-\theta_{1} \theta_{2} s-\theta_{2}^{2}\right) \frac{d^{2} Z(s)}{d s^{2}} \\
-2 \theta_{2} \theta_{3}=0
\end{array}
$$

Thanks to (17), after dividing the expression above by $s^{6}$, we have the result in the time domain:

$$
\begin{aligned}
\theta_{3}=\frac{1}{2 \theta_{2} t^{2}} \int_{0}^{1}\left(\left(2 w_{5,1}-5 w_{4,2}\right) \theta_{2}^{2} t^{4}\right. & +\left(-w_{5,0}-10 w_{3,2}+10 w_{4,1}\right) 2 \theta_{1} \theta_{2} t^{3}+\left(-w_{4,0}+4 w_{3,1}\right) 30 \theta_{2} t^{2} \\
& \left.+120\left(-w_{2,1}+w_{1,2}\right) \theta_{1} t+120\left(-4 w_{1,1}+w_{2,0}+w_{0,2}\right)\right) z(t \tau) d \tau
\end{aligned}
$$

where $w_{m, p}$ denotes $w_{m, p}(\tau)$. The remaining parameter $\theta_{4}$ is estimated in a similar way. In this case, the sets $\Theta_{\text {est }}$ and $\Theta_{\overline{\mathrm{est}}}$ are $\Theta_{\text {est }}=\left\{\theta_{4}\right\}$ and $\Theta_{\overline{\mathrm{est}}}=\left\{\theta_{5}\right\}$. Notice that all already estimated parameters can be used in the estimation of $\theta_{4}$. With respect to the relation $\mathcal{R}$ (see 10), the polynomials $P, Q$ and $\bar{Q}$ for this choice of $\Theta_{\text {est }}$ and $\Theta_{\overline{\mathrm{est}}}$ are $P(s)=s T_{0}(s), Q(s)=s T_{1}(s) \theta_{3}+s T_{2}(s) \theta_{4}$ and $\bar{Q}=T_{0}(s) \theta_{5}$ with $T_{0}(s)=s^{2}+\theta_{1} s+\theta_{2}, T_{1}(s)=s+\theta_{1}$ and $T_{2}(s)=1$. Using Lemma 4 , we obtain a minimal $\bar{Q}$-annihilator w.r.t. $\mathrm{B}_{\Theta}$ given by $\Pi_{\min }^{\Theta}=T_{0}(s) \frac{d}{d s}-T_{0}^{\prime}(s)=\left(s^{2}+s \theta_{1}+\theta_{2}\right) \frac{d}{d s}-\left(2 s-\theta_{1}\right)$. This differential operator applied on polynomials $P, Q$ and $\bar{Q}$ gives:

$$
\begin{aligned}
\Pi_{\min }^{\Theta}(P(s) Z(s)) & =T_{0}(s)^{2}\left(Z(s)+s \frac{d Z(s)}{d s}\right)=\left(s^{4}+2 \theta_{1} s^{3}+\left(2 \theta_{2}+\theta_{1}^{2}\right) s^{2}+2 \theta_{1} \theta_{2} s+\theta_{2}^{2}\right)\left(Z(s)+s \frac{d Z(s)}{d s}\right) \\
\Pi_{\min }^{\Theta}(Q(s)) & =T_{0}^{\prime}(s) \theta_{2} \theta_{3}+\left(T_{0}(s)-s T_{0}^{\prime}(s)\right) \theta_{4}=\left(2 s+\theta_{1}\right) \theta_{2} \theta_{3}+\left(-s^{2}+\theta_{2}\right) \theta_{4} \\
\Pi_{\min }^{\Theta}(\bar{Q}(s)) & =0
\end{aligned}
$$

It results the following algebraic relation $\frac{T_{0}(s)^{2}}{\left(s^{2}-\theta_{2}\right)}\left(Z(s)+s \frac{d Z(s)}{d s}\right)+\frac{\left(2 s+\theta_{1}\right)}{\left(s^{2}-\theta_{2}\right)} \theta_{2} \theta_{3}-\theta_{4}=0$. Thanks to formula (17), after dividing the expression above by $s^{6}$, we have in the time domain:

$$
\begin{aligned}
\theta_{4}= & -t \theta_{2} \theta_{3} \frac{\left(\theta_{1} t+10\right)}{\theta_{2} t^{2}-20}+\frac{1}{t\left(\theta_{2} t^{2}-20\right)} \int_{0}^{1}\left(\left(-w_{5,0}+5 w_{4,1}\right) \theta_{2}^{2} t^{4}+\left(4 w_{3,1}-w_{4,0}\right) 10 \theta_{1} \theta_{2} t^{3}\right. \\
& \left.+\left(3 w_{2,1}-w_{3,0}\right) 20 t^{2}\left(2 \theta_{2}+\theta_{1}^{2}\right)+\left(2 w_{1,1}-w_{2,0}\right) 120 \theta_{1} t-120\left(w_{1,0}-w_{0,1}\right)\right) z(t \tau) d \tau
\end{aligned}
$$

\section{General case}

In the general case, we consider the following equation $x(t)=\sum_{k=1}^{n} \alpha_{k} \exp \left(i \omega_{k} t+\phi_{k}\right)$. 
1) Frequencies estimation: The estimation of frequencies is equivalent to the estimation of all parameters $\left\{\theta_{1}, \ldots, \theta_{n}\right\}$ (Lemma 1). The set of desired parameters is then $\Theta_{\mathrm{est}}=\left\{\theta_{1}, \ldots, \theta_{n}\right\}$ and $\Theta_{\overline{\mathrm{est}}}=\left\{\theta_{n+1}, \ldots, \theta_{2 n+1}\right\}$. Therefore the relation $\mathcal{R}$ (10) is given by

$$
0=P(s) Z(s)+Q(s)+\bar{Q}(s)=s T_{0}(s) Z(s)+\sum_{j=0}^{n-1} T_{j+1}(s) \theta_{n+j+1}+T_{0}(s) \theta_{2 n+1}
$$

The minimal $\bar{Q}$-annihilator provided by Corollary 3 is $\Pi_{\min }=s^{n+1} \frac{d^{n+1}}{d s^{n+1}}$. The action of this annihilator on $P(s) Z(s)$ above gives an algebraic expression depending on $\theta_{1}, \ldots, \theta_{n}$ :

$$
\begin{aligned}
& \Pi_{\min }(P(s) Z(s))=s^{n+1} \sum_{k=0}^{n+1} p_{n+1-k}(s) \frac{d^{n+1-k} Z(s)}{d s^{n+1-k}} \\
& \text { where } p_{n+1-k}(s)=\left(\begin{array}{c}
n+1 \\
k
\end{array}\right) \frac{d^{k} P(s)}{d s^{k}}= \begin{cases}\left(\begin{array}{c}
n+1 \\
k
\end{array}\right)\left(\frac{(n+1) !}{(n-k+1) !} s^{n-k+1}+\sum_{j=k}^{n} \theta_{n-j+1} \frac{j !}{(j-k) !} s^{j-k}\right) & \text { if } 1 \leq k \leq n+1, \\
P(s) & \text { if } k=0 .\end{cases}
\end{aligned}
$$

Furthermore, $\Pi_{\min }(Q(s))=\Pi_{\min }(\bar{Q}(s))=0$. So, (26) gives:

$$
\sum_{k=0}^{n+1} p_{n+1-k}(s) \frac{d^{n+1-k} Z(s)}{d s^{n+1-k}}=0 .
$$

In terms of $\theta_{1}, \ldots, \theta_{n}$, this expression can be written as $\sum_{j=1}^{n} A_{j}(s) \theta_{j}=-B(s)$ with

$$
\begin{aligned}
& A_{j}(s)=\sum_{k=0}^{n+1-j}\left(\begin{array}{c}
n+1 \\
k
\end{array}\right) \frac{(n+1-j) !}{(n+1-j-k) !} s^{2 n+2-j-k} Z^{(n+1-k)}(s), \forall 1 \leq j \leq n, \\
& B(s)=\sum_{k=0}^{n+1}\left(\begin{array}{c}
n+1 \\
k
\end{array}\right) \frac{(n+1) !}{k !} s^{n+1+k} Z^{(k)}(s) .
\end{aligned}
$$

We obtain one equation in $\Theta_{\text {est }}$. However, $n$ independent equations are needed to linearly identify the parameters of this set. The following result show that this is not possible in the operational domain. See Appendix B for a proof.

Theorem 1: There do not exist two $\bar{Q}$-annihilators w.r.t B leading to $n$ independent equations in $\theta_{1}, \ldots, \theta_{n}$. As in the case $n=2$, two different constructions in the time domain provide:

Solution (A): Return to the time domain and convolute the result with $n$ different functions.

Solution (B): Use $n \bar{Q}$-annihilators to obtain $n$ independent equations in the time domain.

We detail below each of the solutions:

(A) Divide (27) by $s^{2 n+3}$, then return to the time domain using (17). This leads to $\sum_{j=1}^{n} a_{j}(t) \theta_{j}=-b(t)$ with

$$
\begin{aligned}
& a_{j}(t)=t^{n+j+2} \int_{0}^{1}\left(\sum_{k=0}^{n+1-j}\left(\begin{array}{c}
n+1 \\
k
\end{array}\right) \frac{(n+1-j) !}{(n+1-j-k) !} \frac{(-1)^{n+1-k}}{(j+k) !} w_{j+k, n+1-k}(\tau)\right) z(t \tau) d \tau, \forall 1 \leq j \leq n \\
& b(t)=t^{n+2} \int_{0}^{1}\left(\sum_{k=0}^{n+1}\left(\begin{array}{c}
n+1 \\
k
\end{array}\right)^{2}(-1)^{k} w_{n+1-k, k}(\tau)\right) z(t \tau) d \tau .
\end{aligned}
$$

Similarly to the two-dimensional case, for $n$ arbitrary functions $g_{1}(t), \ldots, g_{n}(t)$, we obtain:

$$
\sum_{j=1}^{n}\left(g_{i} \star a_{j}\right)(t) \theta_{j}=-\left(g_{i} \star b\right)(t) \text { for } 1 \leq i \leq n .
$$

Denote by $J$ the matrix $\left(\left(g_{i} \star a_{j}\right)(t)\right)_{i, j=1}^{n}$ and by $B$ the $(n \times 1)$-vector $-\left(\left(g_{i} \star b\right)(t)\right)_{i=1}^{n}$. Also denote by $J_{j}$ the matrix obtained from $J$ where the $j$ th-column is replaced by the vector $-B$. Estimations of $\theta_{1}, \ldots, \theta_{n}$ are then given by $\theta_{j}=\frac{\operatorname{det}\left(J_{j}\right)}{\operatorname{det}(J)}$, for $1 \leq j \leq n$.

(B) Recall that all $\bar{Q}$-annihilators are generated by the minimal $\bar{Q}$-annihilator $\Pi_{\min }=s^{n+1} \frac{d^{n+1}}{d s^{n+1}}$. Thus, the elements of a family $\mathscr{F}$ generated by $\Pi_{\min }$ are of the form:

$$
\Pi=\left(\sum_{i=0}^{m} f_{i}(s) \frac{d^{i}}{d s^{i}}\right) \circ \Pi_{\min } \text { with } f_{i}(s) \in \mathbb{C}(s)(1 \leq i \leq m)
$$


The idea is to write an annihilator $\Pi$ in the canonical form:

$$
\Pi=\sum_{i=n+1}^{m} g_{i}(s) \frac{d^{i}}{d s^{i}}, \text { with } g_{i}(s) \in \mathbb{C}(s) \text { for } n+1 \leq i \leq m
$$

A suitable choice of the rational functions $g_{i}$ brings a consistent system of equations in the time domain : set $m=2 n$ and for $1 \leq i \leq 2 n$, set $g_{i}(s)=1$ and $g_{k}(s)=0$, for $k \neq i$. Then, it suffices to solve the resulting system $A(s)\left(\theta_{1} \ldots \theta_{n}\right)^{T}=-B(s)$ expressed in terms of matrices $A(s)=\left(A_{i, j}(s)\right)_{i, j=1}^{n}$ and $B(s)=\left(B_{j, 1}(s)\right)_{j=1}^{n}$ defined by:

$$
\begin{aligned}
& A_{\ell-n, j}(s)=\sum_{k=0}^{n+1-j}\left(\begin{array}{l}
\ell \\
k
\end{array}\right) \frac{(n-j+1) !}{(n-j+1-k) !} s^{n-j+1-k} Z^{(\ell-k)}(s), \\
& B_{\ell-n, 1}(s)=\sum_{k=0}^{n+1}\left(\begin{array}{l}
\ell \\
k
\end{array}\right) \frac{(n+1) !}{(n+1-k) !} s^{n+1-k} Z^{(\ell-k)}(s),
\end{aligned}
$$

for $1 \leq j \leq n$ and $n+1 \leq \ell \leq 2 n$. We divide the expressions above by $s^{-(n+2)}$ to apply the inverse Laplace transform. Hence, in the time domain, we obtain the system $a(t)\left(\theta_{1} \ldots \theta_{n}\right)^{T}=-b(t)$ where the matrices $a(t)=\left(a_{i, j}(t)\right)_{i, j=1}^{n}$ and $b(t)=\left(b_{j, 1}(t)\right)_{j=1}^{n}$ are given by:

$$
\begin{aligned}
& a_{\ell-n, j}(t)=t^{\ell+j+1} \int_{0}^{1}\left(\sum_{k=0}^{n+1-j}\left(\begin{array}{l}
\ell \\
k
\end{array}\right) \frac{(n-j+1) !}{(n-j+1-k) !} \frac{(-1)^{\ell-k}}{(j+k) !} w_{j+k, \ell-k}(\tau)\right) z(t \tau) d \tau, \\
& b_{\ell-n, 1}(t)=t^{\ell+1} \int_{0}^{1}\left(\sum_{k=0}^{n+1}\left(\begin{array}{l}
\ell \\
k
\end{array}\right)\left(\begin{array}{c}
n+1 \\
k
\end{array}\right)(-1)^{\ell-k} w_{k, \ell-k}(\tau)\right) z(t \tau) d \tau,
\end{aligned}
$$

for $1 \leq j \leq n$ and $n+1 \leq \ell \leq 2 n$. Cramer's rule applied on the resulting matrix provides estimations of $\theta_{1}, \ldots, \theta_{n}$.

Remark 3: In the case of a similar parameter estimation problem of a single noisy sinusoidal waveform signal, a consistent system can be found in the operational domain (see [64]).

Example 1: In the case of a sum of three sinusoidal waveform signals, we have $x(t)=\alpha_{1} \exp \left(i \omega_{1} t+\phi_{1}\right)+\alpha_{2} \exp \left(i \omega_{2} t+\phi_{2}\right)+$ $\alpha_{3} \exp \left(i \omega_{3} t+\phi_{3}\right)$. Consider the biased signal $z(t)=x(t)+\beta$. The algebraic method is applied to estimate the parameters $\theta_{1}$, $\ldots, \theta_{6}$ given by

$$
\begin{gathered}
\theta_{1}=-i\left(\omega_{1}+\omega_{2}+\omega_{3}\right), \theta_{2}=-\left(\omega_{1} \omega_{2}+\omega_{1} \omega_{3}+\omega_{2} \omega_{3}\right), \theta_{3}=i \omega_{1} \omega_{2} \omega_{3} \\
z(0)=x(0)+\beta=-\theta_{4}-\theta_{7} \text { and } \dot{z}(0)=-\theta_{5}, z^{(2)}(0)=-\theta_{6} .
\end{gathered}
$$

We are not interested in the estimation the bias $\theta_{7}$. The signal $z(t)$ satisfies (see (5)):

$$
z^{(3)}(t)+\theta_{1} z^{(2)}(t)+\theta_{2} \dot{z}(t)+\theta_{3} z(t)-\theta_{3} \beta=0 .
$$

The above equation reads in the operational domain:

$$
s^{3} Z(s)-z^{(2)}(0)-\dot{z}(0) s-z(0) s^{2}+\theta_{3} Z(s)+\theta_{2}(s Z(s)-z(0))+\theta_{1}\left(s^{2} Z(s)-\dot{z}(0) s-z(0)\right)-\frac{\theta_{3} \beta}{s}=0 .
$$

Using the polynomials $T_{i}$ defined in (8), we obtain a shorter expression:

$$
s T_{0}(s) Z(s)+s\left(T_{1}(s) \theta_{4}+T_{2}(s) \theta_{5}+T_{3}(s) \theta_{6}\right)+T_{0}(s) \theta_{7}=0 .
$$

where $T_{0}(s)=s^{3}+\theta_{1} s^{2}+\theta_{2} s+\theta_{3}, T_{1}(s)=s^{2}+\theta_{1} s+\theta_{2}, T_{2}(s)=s+\theta_{1}$ and $T_{3}(s)=1$. At first, the frequencies are estimated, or equivalently, the parameters $\theta_{1}, \theta_{2}$ and $\theta_{3}$. So we set $\Theta_{\text {est }}=\left\{\theta_{1}, \theta_{2}, \theta_{3}\right\}$ and $\Theta_{\overline{\text { est }}}=\left\{\theta_{4}, \theta_{5}, \theta_{6}, \theta_{7}\right\}$. The relation $\mathcal{R}$ (10) is $0=P(s) Z(s)+Q(s)+\bar{Q}(s)$ with $P(s)=s T_{0}(s), \bar{Q}(s)=s\left(T_{1}(s) \theta_{4}+T_{2}(s) \theta_{5}+T_{3}(s) \theta_{6}\right)+T_{0}(s) \theta_{7}$ and $Q(s)=0$. A minimal $\bar{Q}$-annihilator (see Corollary 3) is $\Pi_{\min }=s^{4} \frac{d^{4}}{d s^{4}}$, then $\Pi_{\min }(P(s) Z(s))=s^{4} \sum_{k=0}^{4} p_{4-k}(s) \frac{d^{4-k} Z(s)}{d s^{4-k}}$ where $p_{4}(s)=P(s)$, $p_{3}(s)=4\left(4 s^{3}+\theta_{1} 3 s^{2}+\theta_{2} 2 s+\theta_{3}\right), p_{2}(s)=6\left(12 s^{2}+\theta_{1} 6 s+\theta_{2} 2\right), p_{1}(s)=4\left(24 s+6 \theta_{1}\right), p_{0}(s)=24$. Moreover, $\Pi_{\min }(Q(s))=$ $\Pi_{\min }(\bar{Q}(s))=0$. Hence:

$$
\sum_{k=0}^{4} p_{4-k}(s) \frac{d^{4-k} Z(s)}{d s^{4-k}}=0 .
$$

Writing this expression in terms of $\theta_{1}, \theta_{2}$ and $\theta_{3}$, we obtain $A_{1}(s) \theta_{1}+A_{2}(s) \theta_{2}+A_{3}(s) \theta_{3}=-B(s)$ with $A_{1}(s)=s^{7} \frac{d^{4} Z}{d s^{4}}+$ $12 s^{6} \frac{d^{3} Z}{d s^{3}}+36 s^{5} \frac{d^{2} Z}{d s^{2}}+24 s^{4} \frac{d Z}{d s}, A_{2}(s)=s^{6} \frac{d^{4} Z}{d s^{4}}+8 s^{5} \frac{d^{3} Z}{d s^{3}}+12 s^{4} \frac{d^{2} Z}{d s^{2}}, A_{3}(s)=s^{5} \frac{d^{4} Z}{d s^{4}}+4 s^{4} \frac{d^{3} Z}{d s^{3}}$ and $B(s)=24 s^{4} Z(s)+96 s^{5} \frac{d Z}{d s}+$ $72 s^{6} \frac{d^{2} Z}{d s^{2}}+16 s^{7} \frac{d^{3} Z}{d s^{3}}+s^{8} \frac{d^{4} Z}{d s^{4}}$.

In the operational domain, linear identification of the parameters can not be accomplished from the above equation (Theorem 1). The solutions described in the general case apply here: 
Solution (A): Return to the time domain and convolute the result with 3 different functions.

Solution (B): Use $\bar{Q}$-annihilators to obtain three independent equations in the time domain.

(A) Divide the expression (29) by $s^{9}$, then return to the time domain. That provides

$$
\begin{gathered}
a_{1}(t) \theta_{1}+a_{2}(t) \theta_{2}+a_{3}(t) \theta_{3}=-b(t) \\
\text { with } \quad \begin{array}{c}
a_{1}(t)=t^{2} \int_{0}^{1}\left(w_{1,4}-6 w_{2,3}+6 w_{3,2}-w_{4,1}\right)(\tau) z(t \tau) d \tau \\
a_{2}(t)=\frac{t}{6} \int_{0}^{1}\left(3 w_{2,4}-8 w_{3,3}+3 w_{4,2}\right)(\tau) z(t \tau) d \tau, a_{3}(t)=\frac{t^{3}}{6} \int_{0}^{1}\left(w_{3,4}(\tau)-w_{4,3}\right)(\tau) z(t \tau) d \tau \\
\text { and } b(t)=\int_{0}^{1}\left(w_{4,0}-16 w_{3,1}+36 w_{2,2}-16 w_{1,3}+w_{0,4}\right)(\tau) z(t \tau) d \tau
\end{array}
\end{gathered}
$$

Choose arbitrary functions $g_{1}(t), g_{2}(t), g_{3}(t)$ and make the convolution: $\left(g_{i} \star a_{1}\right)(t) \theta_{1}+\left(g_{i} \star a_{2}\right)(t) \theta_{2}+\left(g_{i} \star a_{3}\right)(t) \theta_{3}=$ $-\left(g_{i} \star b\right)(t) \quad$ for $1 \leq i \leq 3$. Denote by $\quad J$ the $J$ matrix $\left(\left(g_{i} \star a_{j}\right)(t)\right)_{i, j=1}^{3}$ and by $B$ the $(3 \times 1)$-vector $-\left(\left(g_{i} \star b\right)(t)\right)_{i=1}^{3}$. Also denote by $J_{j}$ the matrix obtained from $J$ where the $j$ th-column is replaced by the vector $-B$. Estimations of $\theta_{1}, \theta_{2}$ and $\theta_{3}$ are then given by $\theta_{j}=\frac{\operatorname{det}\left(J_{j}\right)}{\operatorname{det}(J)}$, for $1 \leq j \leq 3$.

(B) In this case, a minimal $\bar{Q}$-annihilator generating all other annihilators is $\Pi_{\min }=s^{4} \frac{d^{4}}{d s^{4}}$. Write an arbitrary annihilator $\Pi$ in the canonical form $\Pi=g_{4}(s) \frac{d^{4}}{d s^{4}}+g_{5}(s) \frac{d^{5}}{d s^{5}}+g_{6}(s) \frac{d^{6}}{d s^{6}}$. Choose $g_{4}(s)=1, g_{5}(s)=0, g_{6}(s) ; g_{4}(s)=0, g_{5}(s)=1$, $g_{6}(s)=0$ and $g_{4}(s)=0, g_{5}(s)=0, g_{6}(s)=1$ to obtain three equations in the operational domain leading to the following system in the time domain:

$$
t\left(\begin{array}{ccc}
J_{1} & \frac{t}{6} J_{2} & \frac{t^{2}}{6} J_{3} \\
\frac{t}{2} J_{4} & \frac{t^{2}}{6} J_{5} & \frac{t^{3}}{24} J_{6} \\
t^{2} J_{7} & \frac{t^{3}}{4} J_{8} & \frac{t^{4}}{12} J_{9}
\end{array}\right)\left(\begin{array}{c}
\theta_{1} \\
\theta_{2} \\
\theta_{3}
\end{array}\right)=-\left(\begin{array}{c}
J_{10} \\
t J_{11} \\
t^{2} J_{12}
\end{array}\right)
$$

where $w_{m, n}=w_{m, n}(\tau)=(1-\tau)^{m} \tau^{n}$, for $m, n \in \mathbb{N}$ and $J_{i}=\int_{0}^{1} I_{i} z(t \tau) d u$, for $1 \leq i \leq 12$,

$$
\begin{aligned}
& I_{1}=w_{1,4}-6 w_{2,3}+6 w_{3,2}-w_{4,1}, I_{2}=3 w_{2,4}-8 w_{3,3}+3 w_{4,2}, I_{3}=w_{3,4}-w_{4,3} \\
& I_{4}=15 w_{2,4}-2 w_{1,5}-20 w_{3,3}+5 w_{4,2}, I_{5}=10 w_{3,4}-3 w_{2,5}-5 w_{4,3}, I_{6}=5 w_{4,4}-4 w_{3,5} \\
& I_{7}=w_{1,6}-9 w_{2,5}+15 w_{3,4}-5 w_{4,3}, I_{8}=2 w_{2,6}-8 w_{3,5}+5 w_{4,4}, I_{9}=2 w_{3,6}-3 w_{4,5} \\
& I_{10}=w_{4,0}-16 w_{3,1}+36 w_{2,2}-16 w_{1,3}+w_{0,4}, I_{11}=-w_{0,5}+20 w_{1,4}-60 w_{2,3}+40 w_{3,2}-5 w_{4,1}, \\
& I_{12}=w_{0,6}-24 w_{1,5}+90 w_{2,4}-80 w_{3,3}+15 w_{4,2}
\end{aligned}
$$

Now, simply apply Cramer's rule to solve the system and to estimate $\theta_{1}, \theta_{2}$ and $\theta_{3}$.

Let us return to the study of the general case:

2) Amplitudes and phases estimation: It is enough to estimate the remaining parameters $\theta_{n+1}, \ldots, \theta_{2 n}$ to obtain the estimation of the amplitudes $\alpha_{1}, \ldots, \alpha_{n}$, The step-by-step procedure illustrated in the two-dimensional case works again in the general case. We start with the estimation of $\theta_{n+1}$.

- Estimation of $\theta_{n+1}$ : according to Section III, we set $\Theta_{\text {est }}=\left\{\theta_{n+1}\right\}$ and $\Theta_{\overline{\mathrm{est}}}=\left\{\theta_{n+2}, \ldots, \theta_{2 n}\right\}$. The corresponding relation $\mathcal{R}$ (10) gives the following polynomials:

$$
P(s)=s T_{0}(s), Q(s)=s T_{1}(s) \theta_{n+1}, \bar{Q}(s)=s \sum_{j=1}^{n-1} T_{j+1} \theta_{n+j+1}+T_{0}(s) \theta_{2 n+1} .
$$

The parameters $\theta_{1}, \ldots, \theta_{n}$ identified in the previous subsection will be used to estimate $\theta_{n+1}$. The idea to annihilate the polynomial $\bar{Q}$ is to apply a term-by-term elimination, beginning with the lowest degree term, $s T_{n}(s) \theta_{2 n}$. Using Lemma 4 , a minimal annihilator for this polynomial is $\pi_{n}=s \frac{d}{d s}-1$ that applied on $\bar{Q}$ gives

$$
\pi_{n}(\bar{Q})=\sum_{j=1}^{n-2} s^{2} T_{j+1}^{\prime} \theta_{n+j+1}+\pi_{n}\left(T_{0}(s) \theta_{2 n+1}\right) .
$$

In the same way, the lowest degree term of this polynomial is $s^{2} T_{n-1}^{\prime} \theta_{2 n-1}$ and a minimal annihilator will be $\pi_{n-1}=s \frac{d}{d s}-2$. And so on, let us denote by $\Pi$ the annihilator:

$$
\Pi=\pi_{2} \circ \cdots \circ \pi_{n-1} \circ \pi_{n}, \quad \text { with } \pi_{i}=s \frac{d}{d s}-(n-i+1), 2 \leq i \leq n
$$


that eliminates the coefficients of $\theta_{n+2}, \ldots, \theta_{2 n}$ in $\bar{Q}$. The action of $\Pi$ on $P(s) Z(s)$ gives $\Pi(P Z)=\sum_{r=0}^{n} C_{r} Z^{(r)}(s)$ where $C_{r}(s)=\sum_{k=r}^{n-1} \mathbf{s}(n, k+1) s^{k}\left(\begin{array}{l}k \\ r\end{array}\right) P^{(k)}(s)$, for all $0 \leq r \leq n-1$. It remains to eliminate the term in $\theta_{2 n+1}$, namely $\Pi\left(T_{0}\right)$. Using the notation $R=\Pi\left(T_{0}\right)$ and Corollary 3 , we set:

$$
\widetilde{\Pi}=\left(R \frac{d}{d s}-\frac{d R}{d s}\right) \circ \Pi
$$

This operator clearly eliminates $\bar{Q}$. The next step is to apply it on the polynomial $Q$ :

$$
\widetilde{\Pi}(Q)=(-1)^{n+1}(n-1) ! \theta_{n} \theta_{n+1} \frac{d R}{d s} .
$$

As a result, the operator $\widetilde{\Pi}$ applied on the relation $\mathcal{R}$ gives:

$$
0=\widetilde{\Pi}(P(s) Z(s))+\widetilde{\Pi}(Q)=\sum_{r=0}^{n} \widetilde{C}_{r}(s) \frac{d^{r} Z}{d s^{r}}+(-1)^{n+1}(n-1) ! \theta_{n} \theta_{n+1} \frac{d R}{d s}
$$

where $\widetilde{C}_{0}(s)=R \frac{d C_{0}}{d s}-\frac{d R}{d s} C_{0}, \widetilde{C}_{n}=R C_{n-1}$ and for $1 \leq r \leq n-1, \widetilde{C}_{r}(s)=R\left(\frac{d C_{r}}{d s}+C_{r-1}\right)-\frac{d R}{d s} C_{r}$. The term on the right hand side has degree $2 n-1$. So, to apply the Laplace inverse transform formula, we divide by $s^{2 n}$. That provides the estimation of $\theta_{n+1}$ :

$$
\theta_{n+1}=(-1)^{n} t^{2 n-1} \frac{(2 n-1) !}{(n-1) !} \sum_{r=0}^{n} \mathcal{L}^{-1}\left(\frac{C_{r}(s) Z^{(r)}(s)}{s^{2 n}}\right) .
$$

- Estimation of $\theta_{n+1+\ell}$ for $\ell=0, \ldots, n-1$ : we fix $\ell$ in $\{0, \ldots, n-1\}$ and assume that $\theta_{1}, \ldots, \theta_{n}, \theta_{n+1}, \ldots, \theta_{n+\ell}$ are identified. To estimate $\theta_{n+1+\ell}$, we consider $\Theta_{\mathrm{est}}=\left\{\theta_{n+1+\ell}\right\}$ and $\Theta_{\overline{\mathrm{est}}}=\left\{\theta_{n+2+\ell}, \ldots, \theta_{2 n}\right\}$. The corresponding relation $\mathcal{R}$ (10) gives the following polynomials:

$$
P(s)=s T_{0}(s), Q(s)=s \sum_{j=0}^{\ell} T_{j+1}(s) \theta_{n+j+1}, \bar{Q}(s)=s \sum_{j=\ell 1}^{n-1} T_{j+1} \theta_{n+j+1}+T_{0}(s) \theta_{2 n+1} .
$$

The parameters $\theta_{1}, \ldots, \theta_{n+\ell}$ will be used to estimate $\theta_{n+1+\ell}$. The idea again is to annihilate $\bar{Q}$ by a term-by-term elimination, starting with the lowest degree term, $s T_{n}(s) \theta_{2 n}$. Let us denote by $\Pi_{\ell}$ the annihilator:

$$
\Pi_{\ell}=\pi_{\ell+2} \circ \cdots \circ \pi_{n-1} \circ \pi_{n}, \text { if } 0 \leq \ell \leq n-2
$$

where $\pi_{i}=s \frac{d}{d s}-(n-i+1), \ell+2 \leq i \leq n$ and $\Pi_{n-1}=1$. This annihilator eliminates the coefficients of $\theta_{n+\ell+2}, \ldots, \theta_{2 n}$ in $\bar{Q}$. Using Lemma 3 , we have :

$$
\Pi_{\ell}=\sum_{j=0}^{n-\ell-1} \sum_{k=j}^{n-\ell-1} \mathbf{s}(n-\ell, k+1) \mathbf{S}(k, j) s^{j} \frac{d^{j}}{d s^{j}}
$$

and $\Pi_{n-1}=1$. The action of $\Pi_{\ell}$ on $P(s) Z(s)$ gives $\Pi(P Z)=\sum_{r=0}^{n-\ell-1} C_{r, \ell} Z^{(r)}(s)$ where $C_{r, \ell}$ depends on $s$, $C_{r, \ell}(s)=\sum_{j=r}^{n-\ell-1} \sum_{k=j}^{n-\ell-1} \mathbf{s}(n-\ell, k+1) \mathbf{S}(k, j) s^{j}\left(\begin{array}{l}j \\ r\end{array}\right) P^{(j-r)}(s), \forall 0 \leq r \leq n-\ell-1$. On the polynomial $Q$, we have

$$
\Pi_{\ell}(Q)=\Pi_{\ell}\left(T_{\ell}\right) \theta_{n+1+\ell}+(n-\ell-1) !(-1)^{n-\ell} \theta_{n-\ell} \theta_{n+1+\ell}
$$

To complete annihilate $\bar{Q}$, we have to eliminate the remaining term in $\theta_{2 n+1}$, namely $\Pi_{\ell}\left(T_{0}(s)\right) \theta_{2 n+1}$. Setting $R=$ $\Pi_{\ell}\left(T_{0}(s)\right)$, clearly

$$
\widetilde{\Pi}_{\ell}=\left(R \frac{d}{d s}-R^{\prime}\right) \circ \Pi_{\ell}
$$

annihilates $\bar{Q}$. On $Q$, we obtain $\widetilde{\Pi}_{\ell}(Q)=\mathcal{T}_{\ell} \theta_{n+1+\ell}$ where $\mathcal{T}_{\ell}=\Pi_{\ell}\left(T_{0}\right) \frac{d \Pi_{\ell}\left(T_{\ell}\right)}{d s}-\frac{d \Pi_{\ell}\left(T_{0}\right)}{d s}\left(\Pi_{\ell}\left(T_{\ell}\right)+(-1)^{n-\ell}(n-\ell-1) ! \theta_{n-\ell}\right)$. Therefore, the operator $\widetilde{\Pi}_{\ell}$ applied on the relation $\mathcal{R}$ gives:

$$
0=\widetilde{\Pi}_{\ell}(P(s) Z(s))+\widetilde{\Pi}_{\ell}(Q)=\sum_{0}^{n} \widetilde{C}_{r, \ell}(s) \frac{d^{r} Z}{d s^{r}}+\mathcal{T}_{\ell} \theta_{n+1+\ell}
$$

where $\widetilde{C}_{0, \ell}(s)=R \frac{d C_{0, \ell}}{d s}-\frac{d R}{d s} C_{0, \ell}, \widetilde{C}_{n, \ell}=R C_{n-1, \ell}$ and $\widetilde{C}_{r, \ell}(s)=R\left(\frac{d C_{r, \ell}}{d s}+C_{r-1, \ell}\right)-\frac{d R}{d s} C_{r, \ell}$ for $1 \leq r \leq n-1$. The term on the right hand side has degree $2 n+1$. So, to apply the Laplace inverse transform formula, we divide by $s^{2 n+2}$. That provides the estimation of $\theta_{n+\ell+1}$. 
Example 2: We return to the example $n=3$. The estimation of amplitudes $\alpha_{1}, \alpha_{2}, \alpha_{3}$ and phases $\phi_{1}, \phi_{2}, \phi_{3}$ is equivalent to the estimation of $\theta_{4}, \theta_{5}$ and $\theta_{6}$ (see Lemma 1). We begin by estimating $\theta_{4}$, then $\theta_{5}$ and finally $\theta_{6}$. Hence, we first set $\Theta_{\text {est }}=\left\{\theta_{4}\right\}$ and $\Theta_{\overline{\text { est }}}=\left\{\theta_{5}, \theta_{6}\right\}$. The estimated values for $\theta_{1}$ and $\theta_{2}$ and $\theta_{3}$ can be used in the estimation of $\theta_{4}$. The polynomials $P, Q$ and $\bar{Q}$ in relation $\mathcal{R}$ (see (10)) are:

$$
P(s)=s T_{0}(s), \quad Q(s)=s T_{1}(s) \theta_{4} \quad \text { and } \bar{Q}(s)=s T_{2}(s) \theta_{5}+s T_{3}(s) \theta_{6}+T_{0}(s) \theta_{7},
$$

with $T_{0}(s)=s^{3}+\theta_{1} s^{2}+\theta_{2} s+\theta_{3}, T_{1}(s)=s^{2}+\theta_{1} s+\theta_{2}, T_{2}(s)=s+\theta_{1}$ and $T_{3}(s)=1$. Earlier, we could linearly identify $\theta_{1}, \theta_{2}$ and $\theta_{3}$. But, Theorem 1 shows that is not possible to identify linearly $\theta_{4}, \theta_{5}$ and $\theta_{6}$, so we will use nonlinear equations in $\theta_{1}$, $\theta_{2}$ and $\theta_{3}$. Previous subsection's results indicate that a minimal $\bar{Q}$-annihilator w.r.t. B $\Theta_{\Theta}$ written in the canonical form is:

$$
\Pi_{\min }^{\Theta}=-12 s^{2}+12 s^{3} \frac{d}{d s}-6 s^{4} \frac{d^{2}}{d s^{2}}+\left(2 \theta_{3} s^{2}+2 s^{5}\right) \frac{d^{3}}{d s^{3}}
$$

This differential operator is applied on the relation $\mathcal{R}$, according to the formulas presented in this section. Thanks to formula (17) applied on the resulting algebraic relations, we obtain:

$$
\begin{aligned}
\theta_{4} & =-\frac{1}{6 \theta_{3} t^{3}} \int_{0}^{1}\left(\left(-7 w_{6,3}+3 w_{7,2}\right) \theta_{3}^{2} t^{6}+\left(7 w_{6,2}-7 w_{5,3}-w_{7,1}\right) 6 \theta_{2} \theta_{3} t^{5}+\left(w_{7,0}+63 w_{5,2}-21 w_{6,1}-35 w_{4,3}\right) 6 \theta_{1} \theta_{3} t^{4}\right. \\
& +\left(-9 w_{5,1}+w_{6,0}-10 w_{3,3}+15 w_{4,2}\right) 168 \theta_{3} t^{3}+\left(-w_{2,3}+w_{3,2}\right) 2520 \theta_{2} t^{2}+\left(-w_{3,1}+3 w_{2,2}-w_{1,3}\right) 5040 \theta_{1} t \\
& \left.+5040\left(w_{3,0}+9 w_{1,2}-9 w_{2,1}-w_{0,3}\right)\right) z(t \tau) d \tau
\end{aligned}
$$

Using the same procedure, we estimate $\theta_{4}$ : now, we have $\Theta_{\text {est }}=\left\{\theta_{5}\right\}$ and $\Theta_{\overline{\text { est }}}=\left\{\theta_{6}, \theta_{7}\right\}$. Notice that all already estimated parameters can be used in the estimation of $\theta_{5}$. A minimal $\bar{Q}$-annihilator w.r.t. B ${ }_{\Theta}$ is:

$$
\Pi_{\min }^{\Theta}=6 s^{2}+2 s \theta_{1}+\left(-2 \theta_{1} s^{2}-6 s^{3}\right) \frac{d}{d s}+\left(2 s^{4}-s \theta_{3}+\theta_{1} s^{3}\right) \frac{d^{2}}{d s^{2}}
$$

Applied on relation $\mathcal{R}$, it provides an algebraic equation that we solve for $\theta_{5}$ :

$$
\begin{aligned}
& \theta_{5}=\frac{1}{2 t\left(\theta_{3} t^{3}+210\right)} \int_{0}^{1}\left(\left(2 w_{7,1}-7 w_{6,2}\right) \theta_{3}^{2} t^{6}+\left(-w_{7,0}+14 w_{6,1}-21 w_{5,2}\right) 2 \theta_{2} \theta_{3} t^{5}+\left(6 w_{5,1}-w_{6,0}\right) 42 \theta_{1} \theta_{3} t^{4}\right. \\
& +\left(\left(25 w_{4,1}-6 w_{5,0}+10 w_{3,2}\right) \theta_{3}+\left(2 w_{3,2}-w_{4,1}\right) 5 \theta_{1} \theta_{2}\right) 84 t^{3}+\left(\left(-4 w_{3,1}+12 w_{2,2}-w_{4,0}\right) \theta_{2}+\right. \\
& \left.\left.\left(6 w_{2,2}-8 w_{3,1}+w_{4,0}\right) \theta_{1}^{2}\right) 420 t^{2}+\left(3 w_{1,2}-6 w_{2,1}+w_{3,0}\right) 5040 \theta_{1} t+5040\left(-10 w_{1,1}+2 w_{0,2}+3 w_{2,0}\right)\right) z(t \tau) d \tau
\end{aligned}
$$

Finally, $\theta_{6}$ is identified by the same procedure:

$$
\begin{aligned}
\theta_{6} & =-\frac{1}{t^{2}\left(\theta_{3} t^{3}-42 \theta_{1} t-420\right)} \int_{0}^{1}\left(\left(w_{7,0}-7 w_{6,1}\right) \theta_{3}^{2} t^{6}+\left(w_{6,0}-6 w_{5,1}\right) 14 \theta_{2} \theta_{3} t^{5}+\left(w_{5,0}-5 w_{4,1}\right)\left(2 \theta_{1} \theta_{3}+\theta_{2}^{2}\right) 42 t^{4}\right. \\
& \left.+\left(w_{4,0}-4 w_{3,1}\right)\left(\theta_{3}+\theta_{1} \theta_{2}\right) 420 t^{3}+\left(w_{3,0}-3 w_{2,1}\right)\left(2 \theta_{2}+\theta_{1}^{2}\right) 840 t^{2}+\left(w_{2,0}-2 w_{1,1}\right) 5040 \theta_{1} t+5040\left(w_{1,0}-w_{0,1}\right)\right) z(t \tau) d \tau
\end{aligned}
$$

\section{Simulations}

We present in this Section, the estimation of parameters $\theta_{i}(1 \leq i \leq 2 n)$ in the cases $n=1$ and 2 . The figures below show the results obtained by normalized mean values and variances. The simulation results show the estimation of each parameter versus the estimation time. Each point represents an average of the results over a certain number $N$ of trials.

The noisy signal used for the simulation is illustrated for each $n$, the signal-to-noise ratio is indicated as well. The constant bias $\beta$ is considered to be equal to 7.89 .

For a comparison reference, we use the modified Prony's method (PM) displayed on the graphics by a dot-dashed line. The solid line curve represent the parameter estimation resulting from our algebraic method. Assuming that the perturbation $\beta$ is zero, the comparison results show that the proposed method is more robust to the noise corruption. The figures displaying the corresponding variances confirm our assumption.

\section{REFERENCES}

[1] Montojo J, Milstein L. Channel estimation for non-ideal ofdm systems. IEEE Trans. Commun. Jan 2010; 58(1):146 -156.

[2] Neves A, Mboup M, Fliess M. An algebraic receiver for full response CPM demodulation. International Telecommunications Symposium (ITS 2006), Fortaleza-CE, Brésil, 2006.

[3] Klapuri A. Multiple fundamental frequency estimation based on harmonicity and spectral smoothness. IEEE Trans. Acoust., Speech, Signal Processing Nov 2003; 11(6):804-816.

[4] Karimi H, Karimi-Ghartemani M, Iravani M. Estimation of frequency and its rate of change for applications in power systems. IEEE Trans. Power Delivery 2004; 19(2):472-480.

[5] Wu J, Long J, Wang J. High-accuracy, wide-range frequency estimation methods for power system signals under non-sinusoidal conditions. IEEE Trans. Power Delivery 2005; 20(1):366-374.

[6] Yang J, Liu C. A precise calculation of power system frequency. IEEE Trans. Power Delivery 2001; 16(3):361-366.

[7] Begovic M, Djuric P, Dunlap S, Phadke A. Frequency tracking in power networks in the presence of harmonics. IEEE Trans. Power Delivery April $1993 ; 8(2): 480-486$ 

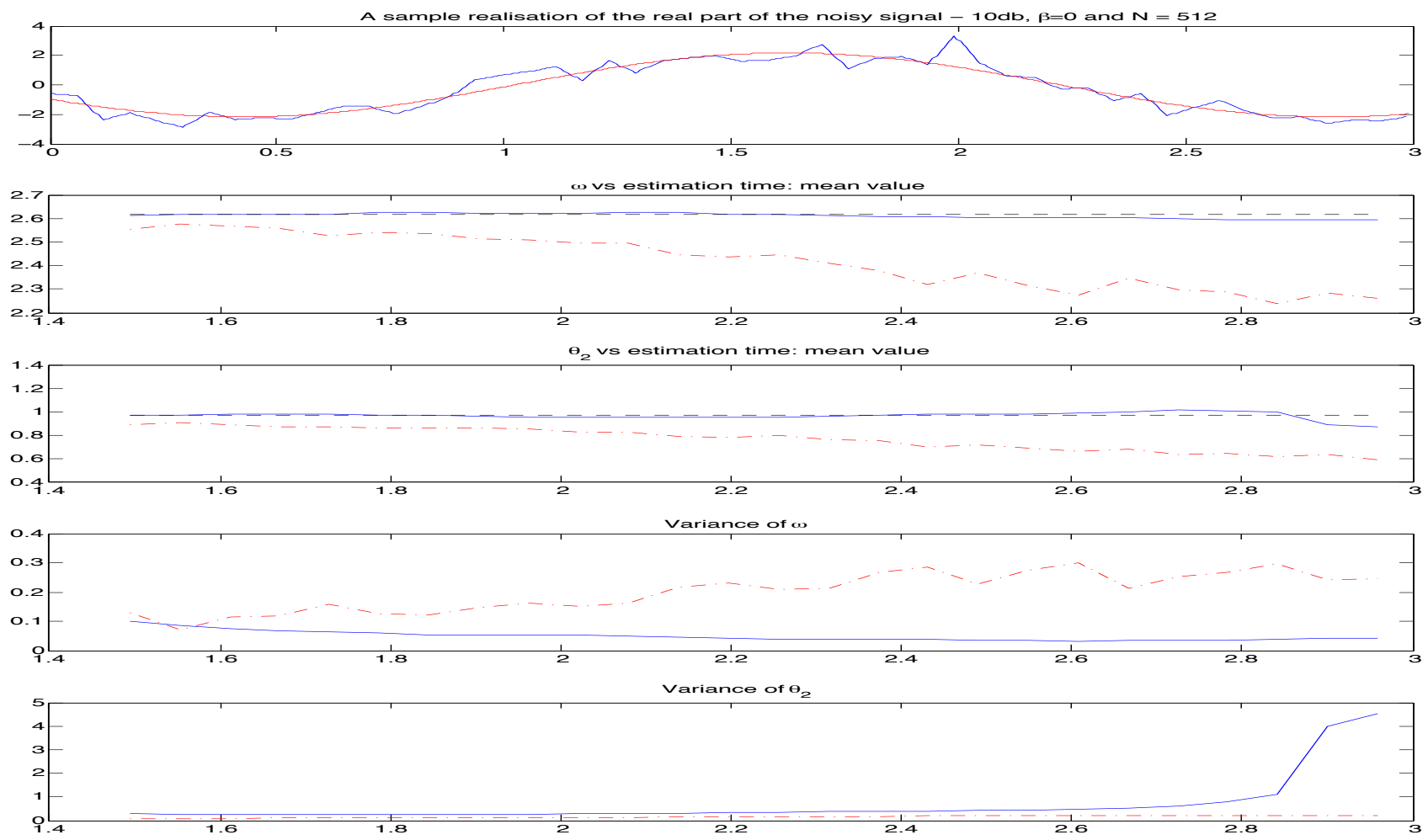

Fig. 1. Case $n=1$ : comparison with the modified Prony's method, $\beta=0$

[8] Östlund N, Yu J, Karlsson J. Improved maximum frequency estimation with application to instantaneous mean frequency estimation of surface electromyography. IEEE Trans. Biomed. Eng. 2004; 51(9):1541-1546.

[9] Zarowski C, Kropyvnytskyy I. Some algorithms for circadian rhythm identification. Proc. IEEE 2001 Pacific Rim Conf. on Communications, Computers and Signal Processing, vol. 2, 2001; $425-428$.

[10] Trapero J, Sira-Ramírez H, Battle V. A fast on-line frequency estimator of lightly damped vibrations in flexible structures. J. Sound Vibration 2007; 307:365-378.

[11] Becedas J, Trapero J, Feliu V, Sira-Ramírez H. Adaptive controller for single-link flexible manipulators based on algebraic identification and generalized proportional integral control. IEEE Trans. Syst., Man, Cybern. 2009; 39(3):735-751.

[12] Hall S, Wereley N. Performance of higher harmonic control algorithms for helicopter vibration reduction. J. Guidance Contr. Dynam. 1993; 116(4):793797.

[13] Bodson M, Douglas S. Adaptive algorithms for the rejection of sinusoidal disturbances with unknown frequency. Proc. 13th IFAC World Conf., San Francisco, CA, USA, 1996.

[14] Herzog R, Buhler P, Gahler C, Larsonneur R. Unbalance compensation using generalized notch filters in the multivariable feedback of magnetic bearings. IEEE Trans. Contr. Syst. Technol. Sept 1996; 4:580-586.

[15] Perruquetti W, Bonnet P, Mboup M, Ushirobira R, Fraisse P. An algebraic approach for humane posture estimation in the sagital plane using accelerometer noisy signal. Proc. 51st IEEE Conf. Decision Control, 2012; 6.

[16] Riche de Prony GM. Essai expérimental et analytique : sur les lois de la dilatabilité de fluides élastiques et sur celles de la force expansive de la vapeur de l'eau et de la vapeur de l'alcool à différentes températures. Journal de l'école polytechnique 1795; 1(22):24-76.

[17] Kahn M, Mackisack M, Osborne M, Smyth GK. On the consistency of Prony's method and related algorithms. J. Comput. Graph. Statist. 1992; 1:329-349.

[18] Osborne M, Smyth G. A modified prony algorithm for exponential function fitting. SIAM Journal on Scientific Computing 1995; 16(1):119-138.

[19] Kay SM, S L Marple J. Spectrum analysis-a modern perspective. Proc. IEEE 1981; 69(11):1380-1419.

[20] Stoica P. List of references on spectral line analysis. Signal Processing 1993; 31(3):329 - 340.

[21] Bresler Y, Macovski A. Exact maximum likelihood parameter estimation of superimposed exponential signals in noise. IEEE Trans. Acoust., Speech, Signal Processing Oct 1986; 34(5):1081 - 1089.

[22] So H, Chang F. A generalized weighted linear predictor frequency estimation approach for a complex sinusoid. IEEE Trans. Signal Processing 2006; 54(4):1304-1315.

[23] Haykin S. Adaptive Filter Theory. $2^{\text {nd }}$ ed. edn., Englewood Cliffs, 1991.

[24] Roy R, Kailath T. Esprit-estimation of signal parameters via rotational invariance techniques. IEEE Trans. Acoust., Speech, Signal Processing Jul 1989; 37(7):984-995.

[25] La Scala BF, Bitmead RR, James MR. Conditions for stability of the extended kalman filter and their application to the frequency tracking problem. Math. of Control, Signals, and Systems (MCSS) 1995; 8:1-26.

[26] La Scala B, Bitmead R, Quinn B. An extended kalman filter frequency tracker for high-noise environments. IEEE Trans. Signal Processing Feb 1996; 44(2):431-434.

[27] Bittanti S, Savaresi S. On the parameterization and design of an extended kalman filter frequency tracker. IEEE Trans. Automat. Contr. 2000; 45:1718 $-1724$.

[28] Friedlander B, Smith J. Analysis and performance evaluation of an adaptive notch filter. IEEE Trans. Inform. Theory mar 1984; 30(2):283-295.

[29] Rao D, Kung SY. Adaptive notch filtering for the retrieval of sinusoids in noise. IEEE Trans. Acoust., Speech, Signal Processing Aug 1984; 32(4):791-802.

[30] Stoica P, Nehorai A. Performance analysis of an adaptive notch filter with constrained poles and zeros. IEEE Trans. Acoust., Speech, Signal Processing June 1988; 36(6):911-919. 

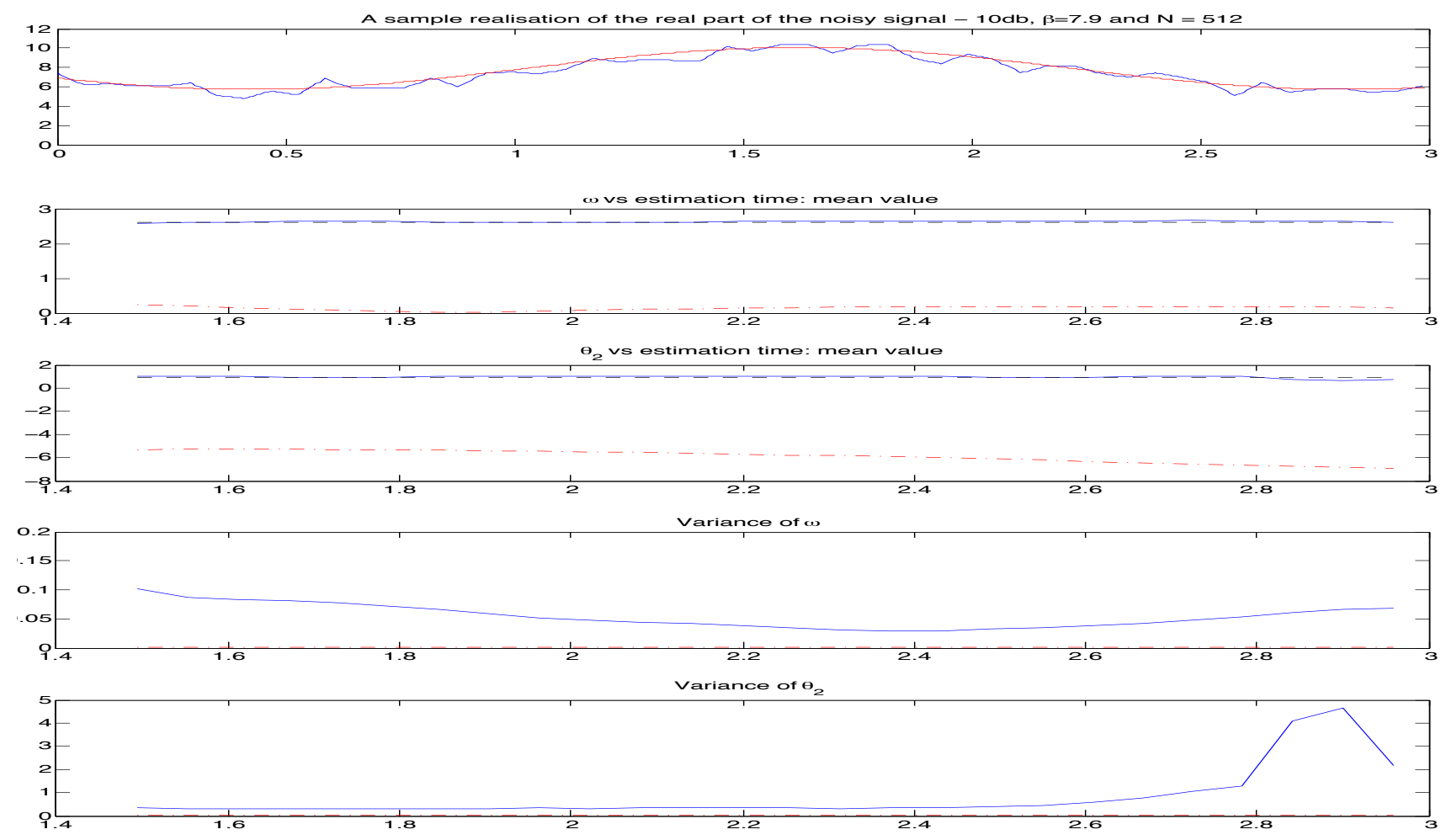

Fig. 2. Case $n=1$ : comparison with the modified Prony's method, $\beta \neq 0$

[31] Bittanti S, Campi M, Savaresi S. Unbiased estimation of a sinusoid in colored noise via adapted notch filters. Automatica 1997; 33:209-215.

[32] Cheng M, Tsai J. A new iir adaptive notch filter. Signal Process 2006; 86:1648-1655. Paris.

[33] Regalia PA. Adaptive IIR Filtering in Signal Processing and Control. Marcel Dekker: New York, 1995.

[34] Fedele G, Ferrise A, Frascino D. Multi-sinusoidal signal estimation by an adaptive sogi-filters bank. 15th IFAC Symp. on Sys. Ident., 2009.

[35] Hsu L, Ortega R, Damm G. A globally convergent frequency estimator. IEEE Trans. Automat. Contr. 1999; 44(4):698-713.

[36] Mojiri M, Bakhshai A. An adaptive notch filter for frequency estimation of a periodic signal. IEEE Trans. Automat. Contr. feb 2004; 49(2):314-318.

[37] Li T, Kedem B. Strong consistency of the contraction mapping method for frequency estimation. IEEE Trans. Inform. Theory 1993; 39(3):989-998.

[38] Liu DJ, Lin CT. Fundamental frequency estimation based on the joint time-frequency analysis of harmonic spectral structure. IEEE Trans. Acoust., Speech, Signal Processing sep 2001; 9(6):609-621.

[39] Bobtsov A, Efimov D, Pyrkin A, Zolghadri A. Switched algorithm for frequency estimation with noise rejection. IEEE Trans. Automat. Contr. sept 2012; 57(9):2400-2404.

[40] Carnevale D, Astolfi A. Hybrid observer for global frequency estimation of saturated signals. IEEE Trans. Automat. Contr. oct 2009; 54(10):2461 -2464.

[41] Carnevale D, Astolfi A. A hybrid observer for frequency estimation of saturated multi-frequency signals. Decision and Control and European Control Conference (CDC-ECC), 2011 50th IEEE Conference on, 2011; 2577-2582.

[42] Xia X. Global frequency estimation using adaptive identifiers. IEEE Trans. Automat. Contr. jul 2002; 47(7):1188-1193.

[43] Coluccio L, Eisinberg A, Fedele G. A property of the elementary symmetric functions on the frequencies of sinusoidal signals. Signal Processing 2009; 89(5):765 - 777.

[44] Mboup M. Parameter estimation via differential algebra and operational culculus. Research report 2007. URL https://hal.inria.fr/inria-00138294.

[45] Hou M. Parameter identification of sinusoids. IEEE Trans. Automat. Contr. February 2012; 57(2):467-472.

[46] Fliess M, Sira-Ramírez H. An algebraic framework for linear identification. ESAIM Control Optim. Calc. Variat. 2003; 9:151-168.

[47] Fliess M, Mboup M, Mounier H, Sira-Ramírez H. Questioning some paradigms of signal processing via concrete examples. in Algebraic Methods in Flatness, Signal Processing and State Estimation, H Sira-Ramírez GSN (ed.). Editiorial Lagares, 2003; 1-21.

[48] Fliess M, Join C, Mboup M, Sira-Ramírez H. Compression différentielle de transitoires bruités. C.R. Acad. Sci. 2004; ser. I (339):821-826.

[49] Fliess M. Critique du rapport signal à bruit en communications numériques. ARIMA 2008; 9:419 - 429.

[50] Fliess M, Sira-Ramírez H. Identification of continuous-time models from sampled data. chapter Closed-loop parametric identification for continuous-time linear systems via new algebraic techniques. Advances in Industrial Control 2008; :362-391.

[51] Fliess M, Join C, Sira-Ramírez H. Non-linear estimation is easy. Int. J. Modelling Identification and Control 2008; 4(1):12-27.

[52] Mboup M. Parameter estimation for signals described by differential equations. Applicable Analysis 2009; 88:29-52.

[53] Fliess M, Join C, Mboup M. Algebraic change-point detection. Applicable Algebra Engin. Communic. Comput. 2010; 21:131-143.

[54] Mboup M, Join C, Fliess M. A revised look at numerical differentiation with an application to nonlinear feedback control. Proc. 15 ${ }^{\text {th }}$ IEEE Medit. Conf. Control Automation - IEEE MED’2007, 2007.

[55] Mboup M, Join C, Fliess M. Numerical differentiation with annihilators in noisy environement. Numerical Algorithms 2009; 50:439-467.

[56] Liu D, Gibaru O, Perruquetti W. Error analysis for a class of numerical differentiator: application to state observation. 48th IEEE Conf. on Decision and Control, Shanghai, China, 2009.

[57] Liu D, Gibaru O, Perruquetti W. Differentiation by integration with jacobi polynomials. J. Comput. Appl. Math. 2011; 235:3015-3032.

[58] Liu D, Gibaru O, Perruquetti W. Error analysis of jacobi derivative estimators for noisy signals. Numerical Algorithms 2011; 50(4):439-467.

[59] Liu D, Gibaru O, Perruquetti W. Convergence rate of the causal jacobi derivative estimator. Lecture Notes in Computer Science 2011; 50(4):439-467.

[60] Trapero JR, Sira-Ramírez H, Battle VF. An algebraic frequency estimator for a biased and noisy sinusoidal signal. Signal Processing 2007; 87(6):1188 $-1201$. 

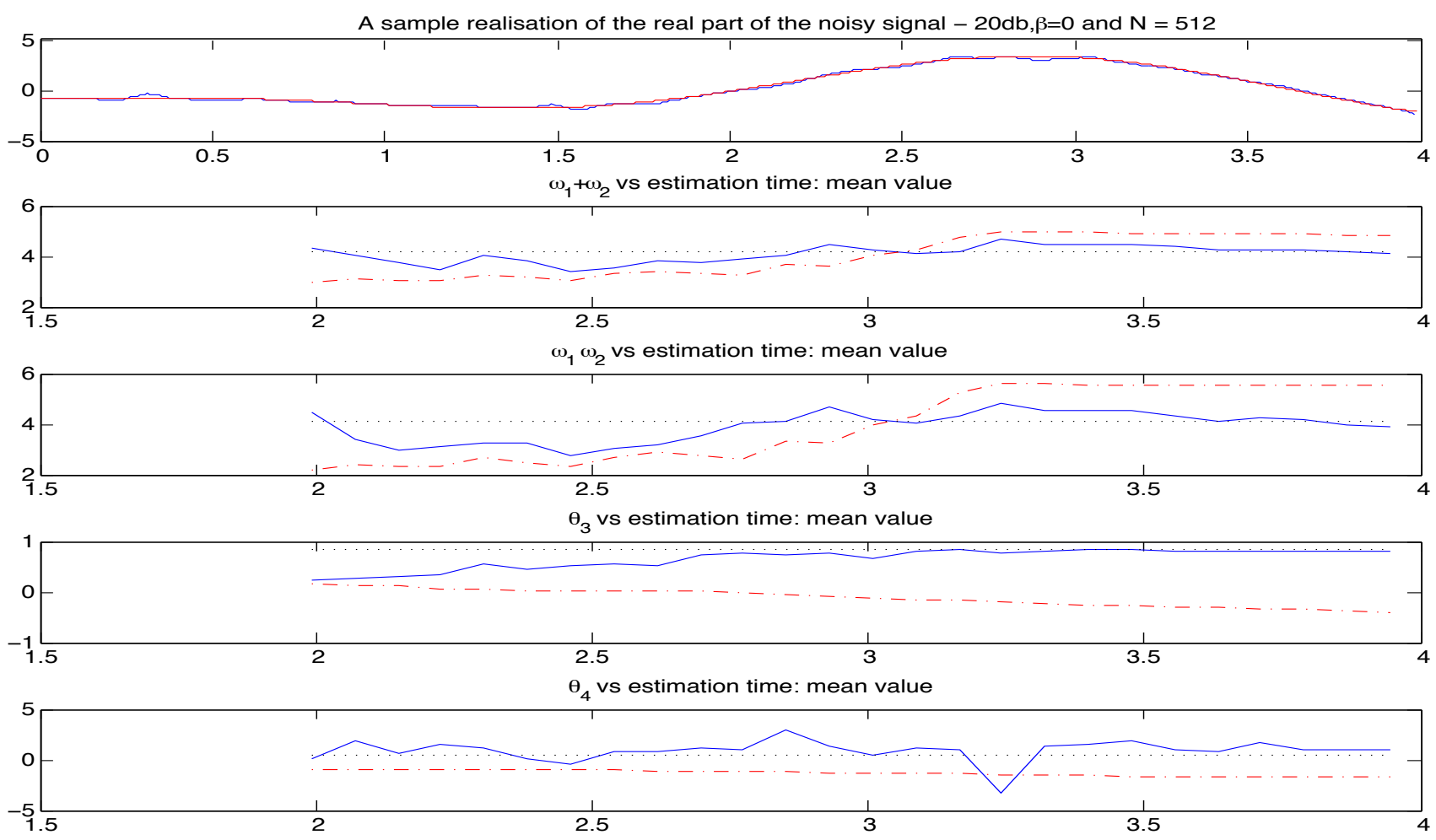

Fig. 3. Case $n=2$ : comparison with the modified Prony's method, $\beta=0$

[61] Trapero-Arenas J, Mboup M, Pereira-Gonzalez E, Feliu V. On-line frequency and damping estimation in a single-link flexible manipulator based on algebraic identification. Control and Automation, 2008 16th Mediterranean Conference on, 2008; 338-343.

[62] Trapero J, Sira-Ramírez H, Batlle V. On the algebraic identification of the frequencies, amplitudes and phases of two sinusoidal signals from their noisy sum. Int. J. Control 2008; 81:507-518.

[63] Liu D, Gibaru O, Perruquetti W, Fliess M, Mboup M. An error analysis in the algebraic estimation of a noisy sinusoidal signal. 16th Mediterranean Conf. on Control and Automation (MED'08), Ajaccio, France, 2008.

[64] Ushirobira R, Perruquetti W, Mboup M, Fliess M. Estimation algébrique des paramètres intrinsèques d'un signal sinusoïdal biaisé en environnement bruité. Proc. Gretsi, Bordeaux, France, 2011.

[65] Ushirobira R, Perruquetti W, Mboup M, Fliess M. Algebraic parameter estimation of a biased sinusoidal waveform signal from noisy data. Sysid 2012, 16th IFAC Symposium on System Identification, Brussels, Belgique, 2012.

[66] Pereira E, Trapero J, Daz I, Feliu V. Adaptive input shaping for manoeuvring flexible structures using an algebraic identification technique. Automatica 2009; 45(4):1046-1051.

[67] Mboup M. Neural spike detection and localisation via volterra filtering. 22nd IEEE Workshop on Machine Learning for Signal Processing, Santander, Spain, 2012.

[68] Dixmier J. Algèbres enveloppantes. Gauthier-Villars, 1974.

[69] McConnell J, Robson J. Noncommutative Noetherian Rings. Hermann, 2000.

\section{APPENDIX}

\section{A. Proof of Lemma 2}

We have $x^{(\ell)}(0)=\sum_{k=1}^{n} \alpha_{k}\left(i \omega_{k}\right)^{\ell} \mathrm{e}^{\left.i \phi_{k}\right)}$. That implies $\left(-\theta_{1}-\theta_{2} \ldots-\theta_{n}\right)^{T}=A\left(\alpha_{1} \mathrm{e}^{i \phi_{1}} \ldots \alpha_{n} \mathrm{e}^{i \phi_{n}}\right)^{T}$ where $A=\left(a_{k \ell}\right)_{k, \ell=0}^{n}$ with $a_{k \ell}=\left(i \omega_{\ell}\right)^{k-1}$ If all frequencies are distinct, then this Vandermonde matrix is invertible and the result follows.

\section{B. Proof of theorem 1}

All $\bar{Q}$-annihilators are of the form $\Pi=\sum_{i=n+1}^{m} g_{i}(s) \frac{d^{i}}{d s^{i}}$, with $g_{i}(s) \in \mathbb{C}(s)$ for $n+1 \leq i \leq m$, for some $m \in \mathbb{N}$. The action of $\Pi$ on $\mathcal{R}(10)$ gives $\Pi(Q)=\Pi(\bar{Q})=0$. Moreover, using proposition 2, we have $\frac{d^{i}}{d s^{i}}(P Z)=\sum_{k=0}^{n+2}\left(\begin{array}{l}i \\ k\end{array}\right) \frac{d^{k} P}{d s^{k}} \frac{d^{i-k} Z}{d s^{i-k}}$ where $P$ has degree $n+1$. Since $\frac{d^{k} P}{d s^{k}}=k !\left(\begin{array}{c}n+1 \\ k\end{array}\right) s^{n+1-k}+\sum_{j=k}^{n} \theta_{n+1-j} k !\left(\begin{array}{l}j \\ k\end{array}\right) s^{j-k}$, it results:

$$
\sum_{i=n+1}^{m} g_{i}(s) \sum_{k=0}^{n+1}\left(\begin{array}{l}
i \\
k
\end{array}\right) k !\left(\left(\begin{array}{c}
n+1 \\
k
\end{array}\right) s^{n+1-k}+\sum_{j=k}^{n} \theta_{n+1-j}\left(\begin{array}{l}
j \\
k
\end{array}\right) s^{j-k}\right) \frac{d^{i-k} Z}{d s^{i-k}}=0
$$




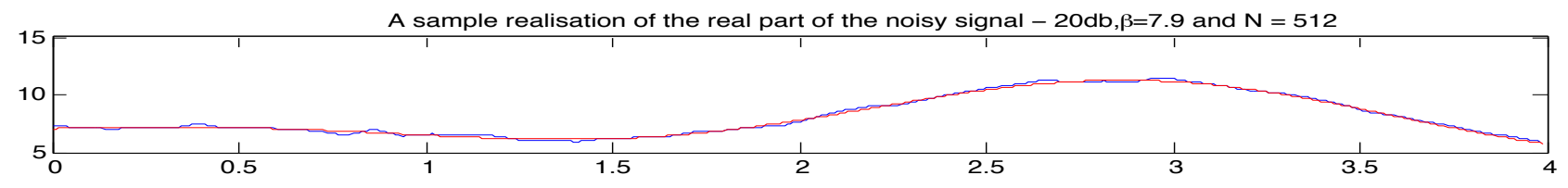

$\omega_{1}+\omega_{2}$ vs estimation time: mean value

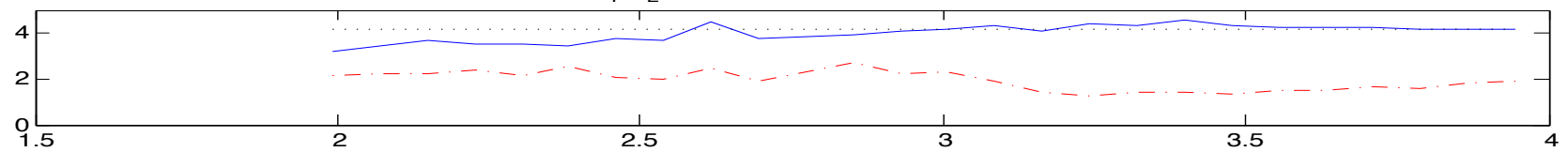

$\omega 1 \omega_{2}$ vs estimation time: mean value

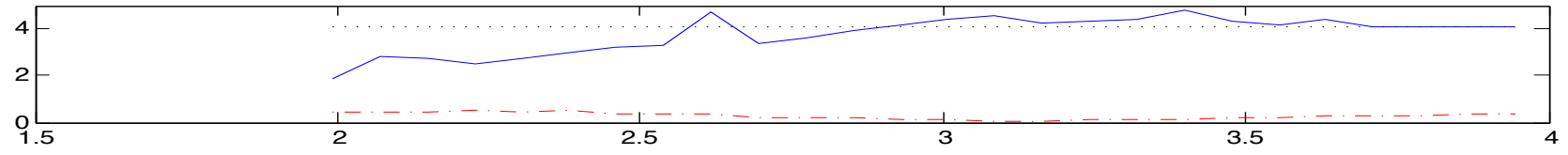

$\theta_{3}$ vs estimation time: mean value

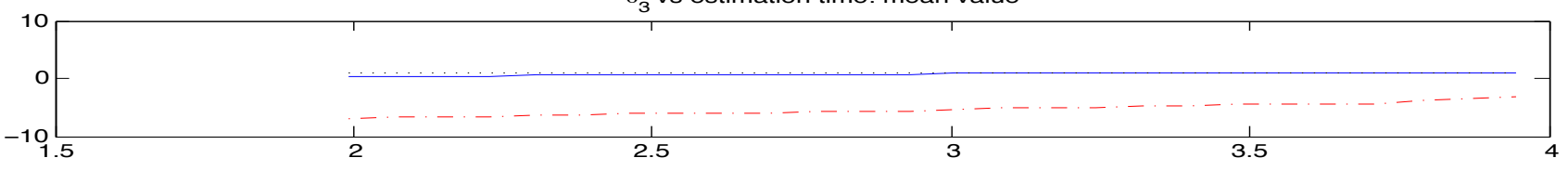

$\theta_{4}$ vs estimation time: mean value

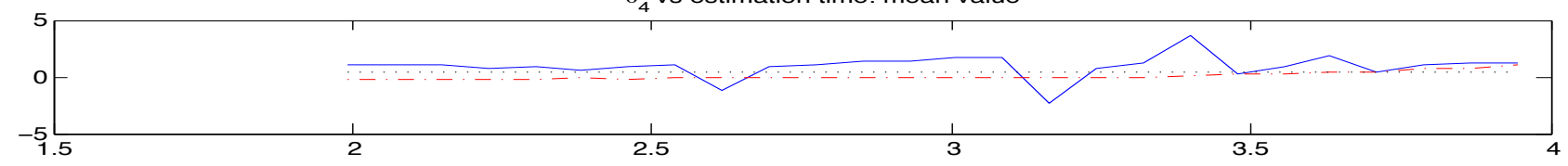

Fig. 4. Case $n=2$ : comparison with the modified Prony's method, $\beta \neq 0$

In the Weyl Algebra framework and using notations in Section IV-A, the formula above reads as $\mathbf{g}^{T}(\widetilde{A} \Theta+\widetilde{B})=0$ where $\mathbf{g}=\left(g_{n+1}(s) \quad \ldots \quad g_{m}(s)\right), \widetilde{A}=\left(\widetilde{A}_{i-n, j}\right)_{i, j}$ is a $(m-n) \times n$-matrix and $\widetilde{B}=\left(\widetilde{B}_{i-n}\right)_{i, j}$ is a $(m-n) \times 1$ vector with

$$
\begin{aligned}
\widetilde{A}_{i-n, j}(s) & =\sum_{k=0}^{n+1-j}\left(\begin{array}{l}
i \\
k
\end{array}\right) k !\left(\begin{array}{c}
n-j+1 \\
k
\end{array}\right) q^{n-j+1-k} p^{i-k}, \quad \text { for } 1 \leq j \leq n \text { and } n+1 \leq i \leq m, \\
\widetilde{B}_{i-n}(s) & =\sum_{k=0}^{n+1}\left(\begin{array}{c}
i \\
k
\end{array}\right) k !\left(\begin{array}{c}
n+1 \\
k
\end{array}\right) q^{n+1-k} p^{i-k}(s) .
\end{aligned}
$$

A somewhat cumbersome computation, that uses relations in Lemma 3, shows that for any $j$, we have $p^{\ell-i} \widetilde{A}_{i-n, j}=\widetilde{A}_{\ell-n, j}$ for $n+1 \leq i \leq \ell \leq m$ and that completes the proof of theorem 1 . 